\title{
LA ZOOLOGÍA EN INTERNET
}

\author{
M. Esteban (*), E. Bello (*) y A. García-Valdecasas (*)
}

\begin{abstract}
Resumen
Se presenta un conjunto de direcciones en Internet (principalmente de páginas World Wide Web), para la búsqueda de documentos y servidores dedicados a la Zoología. Además se hace una referencia exhaustiva a servidores zoológicos por categorías, desde los puramente metodológicos, teóricos, bases de datos, etc. El objetivo es facilitar el uso de esta información online.

Palabras clave: Zoología, biodiversidad, bioinformática, Internet, bases de datos, documentación científica.
\end{abstract}

\section{Abstract \\ Zoology on the Internet}

The main goal of this paper is to facilitate the access of the zoologist to the information online in Internet (mainly through World Wide Web pages). After a presentation of the principal search engines, a number of server address are given for the diferent zoological specialities, from zoological software, data bases, etc. We hope to promote the use of this wealth of information.

Key words: zoology, biodiversity, bioinformatics, Internet, databases, scientific documentation.

\section{Introducción}

Según nuestra experiencia, Internet comienza a ser una fuente imprescindible de información y una herramienta muy práctica en las tareas educativas y de investigación. En el área de Zoología, el número de servidores Internet es enorme y en los últimos años está aumentando de forma exponencial, fundamentalmente gracias al reconocimiento de la importancia que tiene la información en conservación y para el adecuado uso de la biodiversidad (Olivieri et al., 1995; Carling \& Harrison, 1996; Farr \& Rossman, 1997). Las fuentes de esa información son ilimitadas: informes de organizaciones y agencias internacionales, resultados de grupos de trabajo, servicios comerciales de toda índole, pági- nas personales, etc. Además, entre las ventajas de este medio se encuentra la rápidez en la transferencia de información, la libertad de acceso a dicha información para toda la sociedad y la posibilidad de utilización de todo tipo de formatos: texto, imágenes, sonido, etc, con todas las posibilidades del mundo hipermedia. Sin embargo, un uso de este recurso que no vaya dirigido con ciertos criterios estrictos de eficacia, puede ser motivo de pérdidas de tiempo significativas. Dichos criterios exigen ser muy preciso con lo que se busca y cómo se debe buscar. Y esto es así, por varias razones:

- Uno de los objetivos principales de la gran mayoría de los servidores de información que hay en la red, es captar el máximo número de visitantes y orientarles según sus propias directrices, para lo

* Departamento de Biodiversidad y Biología Evolutiva. Museo Nacional de Ciencias Naturales (CSIC). J. Gutiérrez Abascal, 2. Madrid E-28006, España 
cual utilizan infinidad de recursos. Una navegación sin objetivos bien definidos puede fácilmente llevarnos a la deriva, haciéndonos olvidar rápidamente aquello que buscábamos.

- No toda la información en Internet es relevante. Existen muchos servidores de información con un contenido trivial y/o comercial que, debido a cómo responden los "motores de búsqueda de la red" a los perfiles de búsqueda, aparecen seleccionados en paridad a otros servidores más profesionales.

- La información que se encuentra en distintos servidores y hojas es a veces redundante, a lo que hay que añadir la transitoriedad de muchos servidores. Internet es un mundo con "defunciones" y "altas" parecidas a las del mundo real.

- La ausencia de criterios rigurosos para clasificar las hojas, hace que la selección de prioridad en las búsquedas sea una mezcla de presentación y contenido, lo que satisface a pocos usuarios.

- Finalmente, hay que indicar que las comunicaciones en los dias laborables pueden ser muy lentas (el trabajo de búsqueda del presente artículo se ha realizado en gran medida en sábados y domingos).

Por todas estas razones es preciso contar con estrategias de búsqueda para que Internet resulte una herramienta verdaderamente útil de intercambio de información, finalidad para lo que ha sido concebida. El objetivo del presente trabajo es facilitar al zoólogo, y a todas aquellas personas interesadas en este área del conocimiento, sistemas de orientación y búsqueda en las redes internacionales de comunicación. Para ello hemos seleccionado una serie de direcciones y servidores de Internet de especial interés en Zoología, que hemos organizado por temas. Indudablemente esto no va a resolver los problemas derivados de la lentitud de las líneas, pero permitirá llegar a la información requerida de la forma más eficaz.

Existen cientos de direcciones relacionadas con la Zoología — véase Sánchez-Biezma y Aguillo (1997) sólo para Biología Marina-, pero aquí sólo incluiremos servidores de relevancia, tendiendo a primar aquellos que ofrecen información más general sobre los más específicos, ya que estos últimos suelen estar incluidos como enlaces en los primeros. No obstante, se prevee en un plazo breve incluir toda esta información ampliada, actualizada y abierta a sugerencias en el servidor WWW del proyecto Fauna Ibérica (http://www.faunaiberica.museo.csic.es).

Antes de abordar directamente nuestro tema, consideramos útil realizar algunas precisiones de interés general, que nos permitirán introducirnos eficazmente en Internet.
Asumimos que el lector está familiarizado con el concepto básico de Internet y sus herramientas más importantes: Telnet, Ftp y World Wide Web. En el servidor de Ftp anónimo (albia.museo.csic.es/pub/) hay un prontuario de Internet en castellano (manual.exe, en formato autodescomprimible, formato final post-script), que resume los distintos niveles de Internet con direcciones útiles para cada uno de ellos.

A fin de orientarse en materias bibliográficas, sugerimos los siguientes criterios, que a nosotros nos han resultado útiles:

- Las traducciones de libros no son recomendables. Hay que calcular una demora de un año sobre la edición original, y eso en el mundo de Internet es demasiado tiempo.

- Los libros en idiomas originales (preferentemente en inglés) son los más útiles, siempre que hayan sido editados en los últimos seis meses.

- Por norma, los libros escritos por españoles suelen estar al día y con direcciones de ámbito hispano, difíciles de encontrar en otras publicaciones. Para una revisión sobre la bibliografía española sobre Internet consultar Osca-Lluch (1996).

Un problema pendiente y sin solución óptima todavía es como mantener actualizado nuestro registro de direcciones. A diferencia de las bases de datos automatizadas tradicionales, en las que se puede volcar un fichero con un perfil detallado de palabras claves para localizar documentos, Internet adolece todavía de una búsqueda poco eficaz. Hasta la fecha nos permite únicamente utilizar uno o varios términos encadenados mediante operadores lógicos (and, or, not, near, adj, followed by,,+- -). Todavía no se ha llegado a la situación ideal de poder utilizar un perfil de referencia actualizado de forma automática gracias a un robot diana. Sin embargo, estos problemas encontrarán una pronta solución ya que están dentro de los objetivos prioritarios de investigación en el desarrollo de la tecnología en Internet (http://searchenginewatch.com/relevant.htm).

\section{Herramientas de búsqueda}

Antes de entrar de lleno en los servidores de información específicamente zoológicos, nos parece importante comentar la existencia de herramientas generales de búsqueda, como los motores de búsqueda (search engines). Nos ayudan, como herramientas de Internet que son, a conocer los servidores web que pueden contener la información que buscamos, entre los millones de ellos que existen en la actualidad. 
Cada motor de búsqueda tiene su peculiaridad basada fundamentalmente en su rapidez y eficacia para obtener la información. Básicamente funcionan mediante un 'robot', programa especializado, que visita cada página de un web cualquiera cada cierto tiempo y recoge de forma automática la información que contienen. Ésta se almacena por palabras clave o indización de conceptos. Comparan el término a buscar con cada entrada de este índice. Así responden a nuestra pregunta, con un listado de direcciones URL (Universal Resource Locator) que van desde una coincidencia total con los términos de la búsqueda, hasta aproximaciones más generales. Algunos de ellos tienen la posibilidad de poder visualizar, en la respuesta, además de la dirección URL, el título del servidor y una breve descripción del mismo. Como no todos los motores tienen el mismo algoritmo de búsqueda no responden igual a nuestros requerimientos. En ocasiones, es mejor utilizar multibuscadores que mediante una única hoja de cuestionario 'lanzan' nuestra pregunta a varios de los motores de búsqueda.

Existen motores que buscan información general en la red y otros que están organizados en directorios de temas específicos. A éstos podemos añadir los que buscan software, direcciones, etc, en algún servicio de Internet como Archie, Ftp, Gopher, etc.

\section{SisTEMAS DE BÚSQUEDA GENÉRICOS}

Los motores de búsqueda más utilizados se pueden encontrar, entre otras muchas direcciones, en el servidor de la REDIRIS del Consejo Superior de Investigaciones Científicas (http://www.rediris.es/). Uno de los servidores españoles más completos que recoge más de 1.500 direcciones de motores de búsqueda, multibuscadores y buscadores de temas específicos está en la dirección: http://www.prisa.es/ busquedas.htm.

También en Easy Searcher 2 (http://www. easysearcher.com/ez2.html) están los mejores buscadores clasificados por temas. Otras dos direcciones, además de REDIRIS para conseguir otras herramientas de Internet (editores de e-mail, navegadores, etc) gratuitas o shareware son: http://www.uniovi.es/ (Universidad de Oviedo) y http://twcows.arrakis.es/.

A continuación se incluye un listado con los motores de búsqueda más importantes de la red.

\section{En inglés}

\section{AltaVista: $h t t p: / / w w w . a l t a v i s t a . c o m /$}

Es capaz de indizar páginas rápidamente ya que maneja una de las cinco bases de datos de páginas web más importantes y voluminosas, a pesar de ser uno de los últimos en incorporarse a la red. Su pode- roso algoritmo de búsqueda utiliza una forma de cálculo en paralelo, considerado el mejor por facilidad de uso y calidad de resultado (Anónimo, 1997).

C|Net's search.com: $h$ ttp://search.com/

Lanza la pregunta sobre siete motores de búsqueda generales (Yahoo!, Hotbot, etc.), y utiliza motores de búsqueda temáticos organizados jerárquicamente, páginas amarillas, direcciones de correo electrónico, números de teléfono, software, mapas de las ciudades, etc.

Excite: http://www.excite.com/

Permite búsquedas booleanas para concretar más nuestras preguntas.

Hotbot: http://www.hotbot.com/

Es uno de las mejores para encontrar datos de organizaciones, personas, etc. Sus índices son los más voluminosos entre todos los buscadores y realiza un tipo de búsqueda que permite un gran número de términos en cada pregunta.

Infoseek: $h t t p: / / w w w . i n f o s e e k . c o m /$

Una completísima base de datos que indiza el texto completo de páginas web, Ftp y Gopher, direcciones de correo electrónico y FAQs. Aunque en el ranking de las cinco primeras sea la más pequeña, por su gran rapidez en la búsqueda hace que sea una de las más utilizadas.

Lycos: $h t t p: / / w w w . l y c o s . c o m / \mathbf{y}$

LycosPro: $h t t p: / / l y c o s p r o . l y c o s . c o m /$

Lycos es uno de los más utilizados, aunque maneja un volumen de datos inferior a los anteriores. Es uno de los que también indiza ficheros binarios como son los archivos gráficos (GIF, JPEG y MPEG). LycosPro tiene un algoritmo de búsqueda nuevo y más eficaz que además es capaz de recorrer y registrar 10 millones de páginas web al día haciendo actualizaciones semanales de sus bases de datos. No es de los más rápidos pero si exhaustivo. Con los resultados de la búsqueda aparecen un conjunto de términos relacionados con nuestra pregunta que recogen a su vez direcciones de webs.

Magellan: http://www.mckinley.com/

Tiene un directorio donde se puede buscar por temas o bien por palabras clave. Las direcciones web de sus bases de datos se revisan periódicamente.

Net Galaxy: http://galaxy.einet.net/galaxy.html

Utiliza una presentación de búsquedas diferente, ya que muestra grandes temas que después subdivide en términos aproximados. Permite añadir directamente la dirección de nuestro web a su base de datos.

Open Text Index: http://www.opentext.com/

Ofrece muchos datos de los sitios encontrados como URL, título, resumen, fecha y tamaño de cada documento. 
Search: $h t t p: / / w w w . s e a r c h . c o m /$

Multibuscador por temas.

Yahoo!: http://www.yahoo.com/

Buscador estadounidense y podríamos decir que es el más conocido de Internet y, con diferencia, el más visitado. Está organizado por temas como arte, negocios, ciencia, etc. Con Infoseek y Webcrawler es de los pioneros.

Webcrawler: http://webcrawler.com/

Explora más de 440 mil documentos por cada consulta a pesar de lo cual es uno de los que maneja un número menor de documentos. También busca direcciones en sitios Ftp y Gopher.

What's New Too: http://newtoo.manifest.com/

Buscador de los nuevos documentos que aparecen en Internet.

Who's Who on the Internet:

http://web.city.ac.uk/citylive/pages

Busca páginas personales.

En español

B.I.W.E.: http://biwe.cesat.es/

Localiza recursos organizados por temas.

Búscalo rápido:

http://www.enter.net.mx/xyz/atajos/buscalorapido.html

Una buena parte de las páginas de habla hispana de

América puede encontrarse a través de este servidor.

Directorio Global: http://www.dirglobal.net/

Muy bien organizado por temas, con una gran base de datos de webs españoles.

Dónde: http://donde.uji.es

Buscador de recursos de la Universidad Jaume I.

Elcano: $h t t p: / / w w w . e l c a n o . c o m /$

Buscador en castellano. Rápido aunque no muy preciso en sus búsquedas.

Olé: http://www.ole.es/

Servidor de información en castellano muy popular en nuestro país. Principalmente orientado a temas relacionados con cultura, política, etc.

Ozú: http://www.ozu.com/

Otro motor de búsqueda en castellano.

\section{SERVICIOS INTERNET}

Archie: http://www.fwi.uva.nl/fun/archie.html Busca archivos en los Ftp anónimos.

Dejanews: http://www.dejanews.com/

Uno de los más utilizados para localizar páginas de noticias Usenet.

FTP search: http://ftpsearch.unit.no

Localiza Ftp anónimos a nivel mundial. Hytelnet:

http://galaxy.einet.net/hytelnet/START.TXT.html

Para localizar servicios telnet.
LISTZ: http://www.liszt.com/

Busca listados de e-mail y listas de discusión.

\section{Veronica:}

gopher:/veronica.scs.unr.edu/11/veronica

Servicio Veronica que localiza información en Gopher y grupos de noticias.

Wais FAQ Search: $h t t p: / / w w w . c s . r u u . n l /$

Servidor que localiza grupos de noticias y FAQs en la red.

\section{BusCADORES DE RECURSOS EN BIOLOGíA}

Aquatic Ecology Page:

http://www2.netdoor.com/ pinky/aquatic.htm

Interesante servidor de recursos relacionados con los sistemas acuáticos.

Amazing Environmental Organization:

http://www.webdirectory.com/

Uno de los más completos buscadores sobre cuestiones de ecología y medio ambiente.

BIOSCI/Bionet user address database:

http://www.bio.net/addrsearch.html

Utiliza WAIS en las búsquedas y permite operadores booleanos. Localiza direcciones de biólogos en su base de datos.

Cauz: http://www.selu.com/ bio/cauz/links/

En este servidor se encuentran miles de direcciones de páginas web sobre Zoología.

\section{Palette:}

http://www.molbiol.ox. ac.uk/www/ewan/palette.html

En esta página están la mayoría de los buscadores de recursos en biología, tanto gopher como web.

Pedro's Biomolecular Research Tools:

http://www.public.iastate.edu/ pedro/rt_1.html

Buscador de recursos de biología molecular.

Directorios DE RECURSOS EN ZoOLOGíA

Biodiversity and Biological Collections Webserver, The:

http://muse.bio.cornell.edu/

Uno de los servidores pioneros en ofrecer recursos de Zoología con especial énfasis en colecciones. Museums Around the World:

http://www.icom.org/vimp/world.html

Directorio de los museos del mundo organizado por países.

Taxonomy and Systematics at Glasgow:

http://taxonomy.zoology.gla.ac.uk/

Muchos enlaces a sitios relacionados con estas disciplinas.

Zoology Internet Resource Guide:

http://www.york.biosis.org/zrdocs/zoolinfo/zoolinfo.htm

Uno de los mejores servidores en Zoología con la posibilidad de consultar online el 
Zoological Record, información sobre congresos y simposios, directorios de biólogos y taxónomos, programas científicos de ordenador, directorio de páginas web organizado por grupos taxonómicos.

\section{DiRECTORIOS DE TAXÓNOMOS Y CONSERVADORES}

\section{A Who's Who in Studies on Porifera:}

http://www.tcd.ie/People/Bernard.Picton/porifera/ poriwho.html

Listado de nombres, direcciones, e-mail y áreas de interés científico para los biólogos interesados en este grupo.

Arachnology (The study of arachnids):

http://www.ufsia.ac.be/Arachnology/Pages/CIDA.htm

Contiene un listado de direcciones de aracnólogos con enlaces a sus páginas personales y a varios directorios de entomólogos.

Asociation of Systematic Collections:

http://www.ascoll.org/ascdir.html

Con un extenso directorio de socios y personas interesadas en colecciones científicas.

Coleopterists Society:

http://www.auburn.edu/beetles/colememb.htm

Directorio alfabético de los miembros de la sociedad.

\section{Collection Managers online (CMO):}

gopher://gopher.unm.edu:70/00/academic/biology/cmo/ collections.fil

Información muy actualizada con direcciones completas, grupos taxonómicos de estudio, direcciones de correo electrónico, etc., de conservadores, preparadores y demás profesionales americanos e ingleses de la conservación de colecciones.

Directorios de biología:

http://golgi.harvard.edu/afagen/depts/orgs.html

Con numerosos enlaces a sociedades y directorios americanos.

Directorio de biólogos:

http://www.york.biosis.org/zrdocs/desktop/biol-dir.htm

Un directorio con referencias muy interesantes y buenos enlaces a sitios web. En la Zoology Internet Resource Guide.

Directorio de conodontólogos:

http://www.geol.le.ac.uk/staff/map2/pander/Introduction. html\#addresses

Directorio de copepodólogos:

http://www.univaq.it/ sc-amb/wac.html

Listado alfabético con direcciones.

Directorio de dipterólogos americanos:

http://res.agr.ca/ecorc/program2/entomology/diptera/ diptera.htm

Dirección, teléfono, FAX y área de interés.
Directorio de efemeropterólogos:

http://www.famu.edu/mayfly/directory.htm

También es un listado alfabético de direcciones.

Directorio de paleontólogos:

ftp://mailserver.nhm.ac.uk/paleonet/Lists/Doescher Directory

Directorio de quironomidólogos:

http://www.sci.ouc.bc.ca/fwsc/iwalker/intpanis/ director.html

Alrededor de 500 direcciones de taxónomos y sistemáticos interesados en este grupo, con su dirección de correo electrónico y su área de estudio científico.

Directorio de taxónomos:

http://www.york.biosis.org/zrdocs/zoolinfo/syst-tax.htm

Listado de los taxónomos recogidos en el Zoology Internet Resource Guide.

Directorio de taxónomos alemán:

http://www.biologie.uni-uulm.de/biosysdoc/clexpe-1.htm

Listado de direcciones, e-mail y temas de estudio en Alemania.

Directorio de taxónomos italianos:

http://www.univaq.it/ sc-amb/direct.html

Equivalente al anterior para Italia.

DIRTAX (Directorio de Taxónomos de España): http://albia.museo.csic.es/dirtaxhoja.html

Base de datos de los taxónomos españoles organizada jerárquicamente por categorías taxonómicas.

Internationational Research Gropup on Ostracoda:

gopher://Gopher.UH.EDU:70/11/Colleges\%20and\%20 Academics/NSM/About\%20The\%20College\%20of\%20

NSM/6\%20Academic\%20Departments/Geosciences/ IRGO

PALEONET (Directorio de paleontólogos):

ftp://ftp.nhm.ac.uk/paleonet/Lists/

Se puede obtener el directorio completo de paleontólogos a través de Ftp.

Paleovertebrata:

http://eteweb.lscf.ucsb.edu/svp/vpmail

Sociedades científicas:

http://www.lib.uwaterloo.ca/society/biol_soc.html

Servidor exhaustivo con las direcciones web de sociedades científicas, desde microscopía hasta fisiología pasando por ornitología o micología.

Sociedad americana de Nematólogos:

http://ianrwww.unl.edu/ianr/plntpath/nematode/son/son home.htm

Taxonomists of associated collections CONABIO: http://www.conabio.gob.mx/acttax/rechum1.htm

Listado de direcciones de taxónomos e información sobre colecciones. 
TRED The Taxonomic Resources and Expertise Directory: http://www.nbii.gov/tred/

Desarrollado en USA por la Asociation of Systematic Collections, Biological Resources Division, U.S. Geological Survey bajo el liderazgo de Interagency Taxonomic Information System. Tiene una base de datos de 743 expertos en sistemática y taxonomía con información de sus grupos taxonómicos de estudio, habitats y regiones geográficas. Se puede rellenar un cuestionario online y buscar en su base de datos no jerárquica.

UK Systematics Forum Database (UKSF): http://www.nhm.ac.uk/uksf/uksfd/

Se puede obtener información sobre direcciones de contacto, teléfono, etc. mediante preguntas sencillas o múltiples, a partir del grupo taxonómico estudiado, áreas geográficas, instituciones y estratigrafía. World Taxonomists Database ETI:

http://wwweti.eti.bio.uva.nl/database/txnmsts/default.html

Cada taxónomo puede introducir sus datos a través de un formulario. Tiene el inconveniente de no ser una base de datos jerárquica por lo que no permite recuperar datos de los grupos englobados en categorías taxonómicas superiores.

World Directory of Systematic Entomologists: http://nmnhwww.si.edu/gopher-menus/WorldDirectoryof SystematicEntomologists.html

Directorio de entomólogos sistemáticos.

\section{Listas de discusion}

CataList, the official catalog of LISTSERV:

http://www.lsoft.com/lists/listref.html

Desde esta página se puede acceder a casi 15.000 listas publicas de discusión en Internet.

\section{FAQs: Frequently Asked Questions}

\section{FAQs By Category:}

http://www.faqs.org/faqs/by-category.html

Este servidor contiene Usenet Frequently Asked Questions (FAQ) en formato hypertexto y en archivo de texto en Ftp. La información puede obtenerse por categorías, autores, nombres de grupos de noticias, etc.

\section{Zoología por categorías taxonómicas}

La lista de servidores que hacen referencia a alguna de las categorías taxonómicas del Reino Animal es enorme. La mayoría de ellos ofrecen una información que se restringe a áreas geográficas concretas, se ocupan de aspectos muy particulares o están dedicados a niveles taxonómicos cercanos a las categorías de género o especie. Por esta razón, hemos hecho una selección de páginas web que reunen información sobre recursos zoológicos de interés más general, a nivel de filo (siguiendo a Nielsen, 1995) y que cuentan con enlaces a categorías taxonómicas de rango menor.

\section{ZOOLOGÍA GENERAL}

\section{The Tree of Life:}

http://phylogeny.arizona.edu/tree/phylogeny.html

Servidor que contiene información sobre las relaciones filogenéticas y las características de los organismos, ilustra su diversidad y unidad y proporciona enlaces a páginas WWW relacionadas.

\section{Zoology Internet Resource Guide:}

http://www.york.biosis.org/zrdocs/zoolinfo/gp_index.htm

Directorio de páginas web organizado por grupos taxonómicos. Presenta conexiones a los principales servidores de grupos animales por lo que resulta un servidor de referencia.

Museo de Paleontología de la Universidad de California:

http://www.ucmp.berkeley.edu/help/index/

Se trata de un índice que conduce a las páginas web de los diferentes seres vivos organizados por categorías taxonómicas. En cada una de estas páginas obtendremos información sobre sistemática, morfología, registro fósil, etc., además de enlaces a otros recursos relacionados en la red.

\section{PORIFERA}

\section{Porifera Biology on the WWW:}

http://www.tcd.ie/People/Bernard.Picton/porifera/index. html

Se trata de un sitio web que reune un buen número de recursos sobre este phylum.

\section{CNIDARIA \\ Cnidaria WWW Server: http://128.200.5.5/}

Es un servidor de la Universidad de California que reune recursos de interés para el estudio de cualquier aspecto de la biología de los cnidarios.

MolLusCA

Cephalopod Page, The:

http://is.dal.ca/ ceph/wood.html

http://www.vol.it/mirror/cefalopodi/www.html (mirror)

Servidor con muchos recursos para el estudio de los cefalópodos. 


\section{Conchology-Guido T. Poppe:}

http://www.club.innet.be/ year0078/

Guía de recursos muy completa sobre conquiliología.

\section{Eddie's Shell Catalogue:}

http://amsterdam.park.org:8888/Guests/Shells/Shell_Cat alogue//Shell_Pages/Shell_Catalogue_Home_Page.html Catálogo ilustrado de moluscos (conchas).

Information for the malacological community:

http://www.ucmp.berkeley.edu/mologis/mollia.html

Información logística para malacólogos: congresos, revistas, etc., en Internet.

Internet Resources for Conchologists:

http://fly.hiwaay.net/ dwills/shellnet.html

Muchos recursos Internet para conquiliólogos.

Molluscs Net: http://www.molluscs.net/

Servidor muy completo de recursos Internet sobre moluscos.

Scaphopod Page, The:

http://www.hamilton.edu/html/Academic/bio/preynolds/ scaphopodal

Todo sobre los escafópodos.

ANNELIDA

Annelid worm biodiversity resources:

http://www.keil.ukans.edu/ worms/annelid.html

Contiene muchos recursos y conexiones a otros servidores sobre anélidos, poliquetos y oligoquetos, así como a otros grupos: hirudíneos, sipuncúlidos, pogonóforos y vestimentífera.

\section{ARTHROPODA}

Este phylum cuenta con numerosos servidores web. Se listan aquellos que se han considerado más generales, a través de los cuales se accede con más facilidad a servidores de información especializada. Arachnology:

http://www.ufsia.ac.be/Arachnology/Arachnology.html

Todo sobre arácnidos en Internet.

\section{BIOTA databases:}

http://www.sel.barc.usda.gov/database.htm

Bases de datos de los artrópodos terrestres de Norteamérica (no crustáceos).

\section{Crustacean Biodiversity Survey:}

http://www.lam.mus.ca.us/cbs/

Este servidor proporciona un información centralizada de todos los taxa de crustáceos con especial énfasis en el nivel de familia.

Entomology on World-Wide Web (WWW):

http://www.colostate.edu/Depts/Entomology/www_sites. html

Lista de direcciones a sitios relacionados con la entomología.

\section{Entomology Index of Internet Resources:}

http://www.ent.iastate.edu/List/

Un directorio y motor de búsqueda de recursos relacionados con insectos en Internet.

Insects Home Page, The:

http://www.ex.ac.uk/ gjlramel/six.html

Es un buen buscador de información sobre insectos, arácnidos, ácaros y algunos crustáceos.

\section{BRYOZOA}

Bryozoa Home Page, The:

http://www.civgeo.rmit.edu.au/bryozoa/bryozoa.html

Contiene recursos sobre Bryozoa actuales y fósiles.

\section{Nematoda}

\section{Parasitic Nematodes:}

http://ianrwww.unl.edu/ianr/plntpath/nematode/ wormhome.html

Nematodos parásitos de plantas e insectos con muchos recursos en la web.

Wormwood:

http://helios.bto.ed.ac.uk/mbx/fgn/worm/wormhome.html

Servidor orientado a la investigación filogenética de los nematodos.

PHORONIDA

Ascidians pages:

http://160.29.96.22/ascidians/links

Recursos sobre ascidias.

\section{ECHINODERMATA}

\section{Echinoderm Links:}

http://www.calacademy.org/research/izg/echinoderm/ echilink.htm

Listado de enlaces a sitios relacionados con los equinodermos.

\section{VERTEBRATA}

Electronic Resources on Ornithology:

http://www.chebucto.ns.ca/Environment/NHR/bird.html

Direcciones web categorizadas por grandes regiones geográficas.

Herpetology - WWW Virtual Library:

http://cmgm.stanford.edu/ meisen/herp/

Esta página contiene conexiones a todos los sitios conocidos en la red que tratan la herpetología desde el punto de vista científico.

Ichthyology Web Resources:

http://www.biology.ualberta.ca/jackson.hp/iwr/iwr.html

Reúne recursos educativos y científicos organizados en un directorio. Además tiene conexiones a otras páginas que cubren todos los temas relevantes en ictiología. 
MUSE resource search-ictiologia:

http://muse.bio.cornell.edu/cgi-bin/hl?fish

Servidor orientado a colecciones, aunque con interesantes conexiones a otros servidores.

Ornithological Internet Resources:

http://www.bright.net/ vfazio/the-owl.htm

Directorio con un gran número de enlaces a servidores ornitológicos.

Primate:

http://night.primate.wisc.edu/pin/search.html

Base de datos donde se reúnen los principales datos referidos a primates.

World-Wide Web Virtual Library: Fish:

http://actwin.com/WWWVL-Fish.html

Catálogo de conexiones categorizadas por temas

WWW Virtual Library: Whale-Watching-Web: http://www.physics.helsinki.fi/whale/

Servidor que incluye múltiples enlaces relacionados con los cetáceos.

\section{Métodos y herramientas en Zoología}

GENERAL

Biosis:

http://www.biosis.org/htmls/reviews/software.html

Enlaces a más de 150 revisiones de programas informáticos de aplicación en biología. Incluye anatomía, fisiología, taxonomía, etc.

\section{Exeter Software:}

http://members.aol.com/exetersftw/index.html

Compañía comercial de software científico, como NTSYS, MTV, etc., de aplicación en Zoología.

\section{EP-GED Software:}

http://wbar.uta.edu/software/software.htm

Enlaces a programas informáticos de evolución, filogenia y otros.

Checklist of Software for Field Biologists:

http://www.euronet.nl/users/mbleeker/prog/soflis e.html

Referencia general a software de ecología, cartografía, taxonomía, etc.

TAXONOMÍA

An Identification tool for Teachers, Taxonomists and Ecologists:

http://www.ctpm.uq.edu.au/Software/Lucid.html

LucID es una herramienta de identificación para educadores, taxónomos y ecólogos. Permite construir claves, y tiene varias ya terminadas: especies del género Eucalyptus, órdenes de insectos, etc.
Asociación Española de Parques y Jardines

Públicos: http://www.arrakis.es/ jmanuel/linksoft.htm

Asociación Española de Parques y Jardines Públicos, que incluye software sobre plantas ornamentales y de identificación de especies, potencialmente útil para zoólogos.

\section{CABIKEY:}

http://www.cabi.org/catalog/cabikey/cabikey.htm

CABIKEY programa interactivo con claves para los tisanópteros de Europa, varios géneros de mosquitos del mundo, etc. No es shareware.

DELTA (USA):

http://muse.bio.cornell.edu/delta $\mathbf{y}$

DELTA (Australia):

http://www.keil.ukans.edu/delta/www/aust.htm

El paquete DELTA (DEscription Language for Taxonomy) permite construir claves, descripciones naturales y captura de datos por software fenético y filogenético con una única entrada de datos.

Expert Center for Taxonomic Identification (ETI): http://wwweti.eti.bio.uva.nl/

Programa Linnaeus para identificación de varios grupos animales. En CD-ROM.

Multiple Entry Key Algorithm (MEKA):

http://shanana.berkeley.edu/meka/ $\mathbf{y}$

MEKA: http://www.mip.berkeley.edu/meka/

Programa de identificación con múltiples entradas simultáneas.

National Center for Biotechnology Infomation. Taxonomy:

http://www.ncbi.nlm.nih.gov/Taxonomy/taxonomy home.html

Base de datos del NCBI taxonomy con las secuencias de nucleótidos o proteínas de todas las especies que tienen registradas, organizada por categorías taxonómicas.

PANKEY: http://www.rbge.org.uk/pankey.html

PANKEY, conjunto de programas con la filosofía de DELTA y entrada de datos similar. Hubo versiones para Mac. Actualmente en PC-IBM compatibles. Systematics Software for All Lifeforms:

http://www.euronet.nl/users/mbleeker/prog/swtaxlst. html\#ch210

SynKey y SKbrowse software para crear y usar claves sinópticas.

\section{TAXAKEY:}

http://www.cabi.org/catalog/taxakey/taxakey.htm

Clave interactiva de áfidos de cosechas del mundo. No es shareware.

XID: http://www.pullman.com/Business/xid/

XID es un sistema experto de identificación, aplicable a Taxonomía y otras áreas del conocimiento. No shareware. 
FILOGENIA

Journey into Phylogenetic Systematics:

http://www.ucmp.berkeley.edu/clad/clad4.htm

Interesantísimo servidor donde podemos consultar desde conceptos sobre cladismo hasta servidores de software de filogenia.

\section{Phylogenetic programs:}

http://evolution.genetics. washington.edu/phylip/software.html

Servidor general donde se relacionan cerca de 100 programas de filogenia y se dan sus enlaces en Internet. El software está clasificado por metodologías, tipo de ordenador, etc.

\section{Tree of Life:}

http://phylogeny.arizona.edu/tree/life.html

Es uno de los servidores más interesantes para encontrar la información sobre inferencia filogenética, programas y árboles evolutivos más reciente de todos los organismos.

\section{BioestadísticA}

\section{Statistical Power Analysis Software:}

http://www.interchg.ubc.ca/cacb/power/index.html

Lista de programas estadísticos de utilidad en Zoología y Ecología.

\section{Journal of Statistical Software:}

http://www.stat.ucla.edu/journals/jss/

Es una revista online, que revisa software estadístico. Lo mantiene UCLA Statistics. Es gratuita, electrónica y con revisores. En el momento de escribir esto, es más una promesa de futuro, ya que sólo llevan unos pocos números editados.

A Comprehensive Listing of Statistical Software: http://www.statistics.com/vendors.html

Lista de software sobre estadística.

\section{Biostatistics:}

http://www.biostat.washington.edu/Xvlib/

Servidor de Bioestadística, con enlaces.

Species Diversity and Richness:

http://www.irchouse.demon.co.uk/diversity.html

Software para el cálculo de índices de diversidad y su evaluación estadística. No shareware.

\section{MORFOMETRÍA}

Morphometrics at SUNY Stony Brooks:

http://life.bio.sunysb.edu/morph

El servidor de la nueva morfometría geométrica, con programas, documentos y lista de discusión.

\section{BIOCARTOGRAFÍA}

Spatially Oriented Research in Ecology:

http://userzweb.lightspeed.net/ jpthomas/spore3.html

Software para biogeografía, modelos de datos espaciales, etc.
Software for Distribution and Coincidence Mapping:

http://www.dmap.co.uk/

DMAP software. Para análisis de distribuciones, en especial con aplicaciones biológicas. No shareware.

Cartographic Links for Botanists:

http://www.helsinki.fi/kmus/cartogr.html

Cartografía para botánicos, pero muy útil para zoólogos. Muchos enlaces.

\section{ECOLOGíA}

\section{Ecology Software:}

http://nhsbig.inhs.uiuc.edu/www/index.html

Depósito de programas útiles en ecología, evolución y Zoología.

Microcomputer Power:

http://ww1.microcomputerpower.com/webpages/mcp/

Distribuidor de Canoco y otros programas de ecología.

Ecological and Environmental Software:

http://www.ramas.com/

RAMAS. Conjunto de programas para ecología y medio ambiente. No shareware.

\section{BiomATEMÁTICA}

Artificial Life On Line: http://alife.santafe.edu/

Es el más importante de los servidores sobre vida artificial y en él encontramos desde software gratuito hasta las referencias de las publicaciones más recientes sobre el tema.

\section{Biomathematics \& Biostatistics:}

http://www.ability.org.uk/biomath.html

Muchos enlaces a biomatemática y bioestadística.

\section{TÉCNICAS MOLECULARES}

\section{Phylo-win:}

http://acnuc.univ-lyon1.fr/phylogeny/phylowin.html

Phylo-win, programa para inferencia filogenética molecular.

Software Development for Molecular Biology:

http://www.gsf.de/biodv/software.html

Varios programas para biología molecular.

\section{Molecular Biology:}

http://www.ingenlabs.com/molecular.html

Enlaces a múltiples sitios y recursos en biología molecular.

\section{Fuentes de Información Bibliográfica}

Otro servicio de gran utilidad que proporciona Internet es el rápido acceso a la documentación científica. A continuación incluimos una relación 
de servicios ordenados por temas que nos facilitarán esta tarea.

\section{BIBLIOTECAS}

Hoy en día son muy numerosas las bibliotecas que ofrecen servicio a través de Internet y que facilitan el acceso online a sus catálogos, índices y demás servicios. En este apartado hemos seleccionado algunas direcciones de bibliotecas y servidores a través de los cuales podremos obtener información de la mayor parte de las bibliotecas del mundo.

\section{Biblioteca Nacional de España:}

http://www.bne.es/

Además de información sobre los fondos de la Biblioteca Nacional este servidor reune una serie de recursos de interés para bibliotecarios y usuarios: catálogos de librerías, editoriales, asociaciones, etc.

\section{Unidad de Coordinacion de Bibliotecas del CSIC:} http://www.csic.es/cbic/cbic.htm

Informa sobre los servicios que ofrecen las bibliotecas del CSIC: información de la red, catálogos y bases de datos del CSIC, servidores WWW de bibliotecas del CSIC, acceso online a los catálogos de dichas bibliotecas, así como enlaces a otros recursos.

\section{Bibliotecas Universitarias y Científicas:}

http://www.csic.es/cbic/webuni.htm

Página incluida en el servidor de bibliotecas del CSIC que facilita enlaces a las bibliotecas universitarias así como a los catálogos colectivos de las redes de dichas bibliotecas.

Bibliotecas Públicas del Estado:

http://www.mcu.es/bpe/bpe.html

Página del Ministerio de Cultura que permite acceder a los catálogos de las Bibliotecas Públicas del Estado.

Servidores de Bibliotecas en España:

http://www3.uniovi.es/Vicest/Recursos/Spain_OPACS. $h \mathrm{tml}$ (versión imágen activa-mapa)

http://www.rediris.es/recursos/mapas/OPAC/index.html (versión texto).

\section{Bibliotecas Nacionales de Europa:}

http://portico.bl.uk/gabriel/en/eurocoun.html

Listado de las bibliotecas nacionales europeas con datos informativos sobre cada una de ellas y enlaces, cuando existen, a sus servidores.

Directorio de Bibliotecas Españolas e Internacionales:

http://www.unileon.es/dp/abd/bibliot.htm

Directorio online muy completo de bibliotecas españolas y extranjeras con enlaces a todas ellas. Muy recomendable como punto de partida.
WebCATS:

http://library.usask.ca/hywebcat/ Catálogos de Bibliotecas en WWW.

Catálogos de la biblioteca del British Museum of Natural History:

telnet://libcat@library.nhm.ac.uk; login: libcat

Este catálogo online permite búsquedas por autor, título, series y palabras clave.

\section{EDITORIALES Y DISTRIBUIDORES DE LIBROS}

Listados de editoriales académicas y científicas con servicio online en WWW:

Catálogos de editoriales y librerías:

http://dalila.ugr.es/ felix/g2/a5.htm

Colección de enlaces a editoriales con un apartado para editoriales y librerías científicas en España.

WWW Virtual Library: List of online publishers: http://www.comlab.ox.ac.uk/archive/publishers/list.html WWW Virtual Library: Academic Publishers: http://www.edoc.com/jrl-bin/wilma/pac

WWW Virtual Library: Scientific / Technical / Medical (STM) Publishers:

http://www.edoc.com/jrl-bin/wilma/stm

Listado general de editoriales españolas con servicio online en WWW:

Editoriales Españolas:

http://www.bne.es/editesp.htm

Distribuidores especializados en temas de Historia Natural

Amazon: http://www.amazon.com/

Es uno de los distribuidores de libros más importantes de Internet. Cubre todas las especialidades y cuenta con un gran fondo de libros antiguos.

BookWeb: Bookstore Directories:

http://www.bookweb.org/directory/

Directorio de distribuidores americanos de libros con servicio online

Balogh Scientific Books, botany, zoology, ecology, geology books:

http://www.balogh.com/ balogh/catalogs.html

Conchological books:

http://www.dada.net/naturama/conch.html

CSIRO Books - Zoology:

http://www.publish.csiro.au/monographs/bzoo.html

Koeltz Scientific Books:

http://www.koeltz.com/

Natural History Book Service:

http://www.nhbs.co.uk/index.html 
BIBLIOGRAFÍA

$\underline{\text { Servicios }}$ bibliográficos online

Bioline Publications:

http://www.bdt.org.br/bioline/

Se trata de un servicio, orientado exclusivamente a biocientíficos, cuyo fin es facilitar la adquisición de documentación científica. Trabaja en colaboración con la Base de Dados Tropical (BDT) de Brasil.

BIOSIS: $h t t p: / / w w w$.biosis.org/

Esta compañía publica las siguientes bases de datos: BIOSIS Previews, BIOSIS Previews/RN, Zoological Record Online. En la siguientes dirección se pueden encontrar las compañías que ofrecen los sistemas de búsqueda para acceder a ellas: http://www.biosis.org/htmls/vendors/index.html.

BIOSIS/Zoological Record ofrece asimismo la base de datos TRITON de nomenclatura zoológica que puede ser de acceso libre (http://www.york.biosis. org/triton/indexfm.htm) o en una versión comercial más completa.

CatchWord Ltd: http://www.catchword.co.uk

Se trata de una editorial exclusivamente de revistas electrónicas. Estas son editadas en RealPage y son accesibles a través de Internet por suscripción.

Deutsches Institut für Medizinische Dokumentation und Information (DIMDI):

http://www.dimdi.del

Proporciona acceso a las principales bases de datos científicas, principalmente de biomedicina.

European Space Agency Information Retrieval Service (ESA-IRS):

http://www.esrin.esa.it/htdocs/esairs/databases/ subject.html.

Proporciona servicio online sobre información científico técnica aerospacial, pero también incluye bases de datos de biología, ciencias acuáticas, ciencias ambientales etc.

Internet Database Service:

http://csa.sara.nl/ids.html

Servicio por suscripción que proporciona acceso a más de 35 bases de datos publicadas por Cambridge Scientific Abstracts y otras editoriales. Incluye información de ciencias acuáticas, biológicas y ambientales.

\section{Institute for Scientific Information (ISI):}

http://www.isinet.com/

Es un editor de bases de datos que indiza datos bibliográficos, referencias citadas y abstracts de fuentes científicas, técnicas y médicas. Publica Current Contents y Science Citation Index, entre otros.

Knight-Ridder Information-Web-Based Products: http://www.krinfo.com/

Es una compañía comercial que proporciona diferentes servicios de acceso a información en un elevado número de bases de datos que abarcan numerosas disciplinas. A continuación indicamos todos aquellos productos que ofrecen y que permiten acceso a información científica:

DIALOG WEB: http://dialog.krinfo.com/

DIALOG SELECT: http://dialogselect.krinfo.com/

DATASTAR WEB: http://dsweb.krinfo.ch/

SCIENCE BASE: http://krscience.dialog.com/

SOURCE ONE: http://krsourceone.krinfo.com/

OCLC-Online Computer Library Center,

Incorporated: http://www.oclc.org/

Organización sin fines de lucro dedicada a la investigación y a la prestación de servicios computarizados de información. La red y los servicios de OCLC vinculan a más de 25.000 bibliotecas de Estados Unidos y de 63 países más. Dentro de los servicios de consulta ofrecidos por la OCLC de interés para el zoólogo se encuentra el FirstSearch de OCLC con acceso vía WWW a través de http://www.ref.oclc.org:2000/ o mediante http://www.ref.oclc.org/europe/ y también http:// www.ref.uk.oclc.org:2000/ para Europa. Este servicio ofrece más de 60 bases de datos bibliográficas entre las que se encuentran: WorlCat, Article First, ContentsFirst, FastDoc NetFirst, Environmental Sciences \& Pollution, AGRICOLA, General Sciences Abstratcts, GEOBASE, GeoRef, INSPEC, Biological \& Agricultural Index y Chemical Abstracts Services (CA) Student Edition, especializadas en Ciencias en general y en Biología en particular.

STN International: http://www.cas.org/stn.html

Compañía comercial que proporciona acceso a información online en bases de datos sobre Ciencia y Tecnología, entre otras.

UnCoverWeb: $h t t p: / / u n c w e b . c a r l . o r g /$

Servicio por suscripción que facilita regularmente documentación online del contenido de los últimos números de revistas e incluso de los mismos artículos, que se pueden obtener a la vez que aparecen publicados. Cuenta con un fondo de más de 17.000 revistas de habla inglesa de todas las áreas del conocimiento.

\section{UNESCO:}

http://www.unesco.org/general/eng/infoserv/db/index.html

Bases de datos online de los temas de competencia de la UNESCO (educación, ciencias, cultura y comunicación)

\section{WilsonWeb:}

http://www.hwwilson.com/tecfaq7.html

Esta compañía comercial proporciona acceso online a un conjunto de bases de datos que abarcan diferentes áreas del conocimiento. Entre ellas destacamos Biological \& Agricultural Index. 
$\underline{\text { Bases de datos bibliográficas online: }}$

A) Por categorías taxonómicas

1. Porifera

Papyrus Porifera Bibliography:

http://www2.tcd.ie/People/Bernard.Picton/porifera /poribib.html

Base de datos que se puede adquirir a través de la red en formato zip.

2. Cnidaria

Bibliography of Octocorallia from Pliny to the Present:

http://nmnhwww.si.edu/gopher-menus/Octocorals.html

Bibliografía de Octocorallia en servidor gopher.

Coral Health And Monitoring Literature Abstracts:

http://coral.aoml.noaa.gov/bib/lit.abstracts.html

Proporciona resúmenes de revistas y conferencias científicas sobre corales.

3. Mollusca

Biology of abalones, The:

http://www.dada.net/naturama/malafile.html

Puede consultarse online o cargarse en versión para Macintosh o versión texto.

Cephalopod Bibliography Online Service:

http://www.york.biosis.org/zrdocs/zoolinfo/cephbibl.htm

Hawaiian Terrestrial and Freshwater Snails

Bibliography-Gopher Index:

gopher://gopher.bishop.hawaii.org:70/77/index/ biblio.snails

Malacology:

gopher://erato.acnatsci.org:70/77/.wasp/.index/indexbib

Referencias sobre sistemática, biogeografía y diversidad de gasterópodos marinos del oeste del Atlántico, desde Groenlandia hasta la Antártida.

Nudibranch Bibliography:

http://scilib.ucsd.edu/sio/indexes/mcdonald.html

Cubre todas la publicaciones que tratan sobre nudibranquios excepto libros de texto y animales locales marinos.

Opisthobranch Bibliographic Data Search:

http://www.oz.net/miranda/searchpara.html

Scaphopod Bibliographic Database:

http://www.hamilton.edu/html/Academic/bio/ preynolds/scaphopoda/CGI/Scaph.html

Más de 1.200 referencias sobre escafópodos.

\section{Arthropoda}

Almond Moth Bibliography:

http://sun1.oardc.ohio-state.edu/cocoa/almond.htm

Alrededor de 600 referencias sobre Ephestia cautella (Walker).
Bibliography of aquatic Coleoptera:

http://bio-www.tamu.edu/beetles/NABS1996.htm

Referencias sobre coleópteros acuáticos en el año 1996.

Bibliography of attractants of blood feeding arthropods:

gopher://minerva.forestry.umn.edu:70/11/bugs

Bibliografía sobre la tecnología basada en atrayentes para el control de insectos hematófagos.

Bibliography of Crustacea Isopoda:

http://nmnhwww.si.edu/gopher-menus/Isopods.html

Referencias sobre isópodos marinos y de agua dulce de todo el mundo.

California Islands Entomological Bibliography:

gopher://muse.bio.cornell.edu:70/11/project_information/ faunas

Esta bibliografía incluye más de 1.000 citas sobre la entomología de las islas de California.

Crayfish bibliography (Crustacea, Astacura): http://nmnhwww.si.edu/gopher-menus/Crayfish.html

Esta bibliografía contiene más de 13.000 referencias de estos crustáceos de agua dulce .

Cultural Entomology Bibliography:

http://www.insects.org/cebib/aa.html

Referencias sobre la influencia de la entomología en diferentes áreas de la cultura.

Chironomid References:

http://oksw01.okanagan.bc.ca/fwsc/iwalker/intpanis/

Conjunto de bibliografías sobre biología, ecología, paleoecología y sobre el género Chironomus.

Formis ant bibliography (telnet session):

telnet://luis@luis.nerdc.ufl.edu/

FORMIS: An Ant Bibliography

http://www.public.iastate.edu/ entomology/FORMIS/ AboutFORMIS.html

FORMIS es una base de datos que contiene casi 20.000 referencias sobre hormigas

Insect Bibliography Server:

http://129.93.226.138/bibwww/bib.htm

Conjunto de bibliografías sobre diferentes temas y grupos de insectos.

World Dryopoid (Coleoptera) Bibliography:

gopher://CAS.calacademy.org:70/11/depts/ent/wdryopoid/

Referencias sobre Dryopiodea acuáticos y semiacuáticos (Familias Dryopidae, Elmidae, Eulichadidae, Limnichidae, Psephenidae y Ptilodactylidae)

World Mecoptera Bibliography:

gopher://CAS.calacademy.org:70/11/depts/ent/wmecoptera

Referencias sobre Sistemática y Biodiversidad en este orden de insectos.

World Neuropteroid Bibliography:

gopher://CAS.calacademy.org:70/11/depts/ent/wneurop Contiene unas 7.500 referencias sobre este orden. 
5. Nematoda

BiblioFil/OnchoBib:

http://helios.bto.ed.ac.uk/mbx/BiblioFil/bibliofil.html

Archivo de bibliografías sobre filarias.

Nematode Bibliography Server:

http://129.93.226.138/nematode/nemabib.htm

Bibliografías de nematodos entomopatógenos y

de genética de nematodos.

Ditylenchus: http://dawn.fenk.wau.nl/form2.html

Bibliografía sobre este género.

6. Phoronida

Bibliography on Tunicates

http://biol.dgbm.unina.it:8080/ascidians/bibliography.html

Reúne 36 años de citas bibliográficas sobre

Tunicados. Ofrece varios tipos de consulta.

7. Vertebrata

Bibliography of Crocodilian Biology:

http://www.med.jhu.edu/FAE/meers/croc.bib.cover.html

Incluye unas 2.500 referenciass sobre la biología de los cocodrilos, desde la paleontología y la ecología pasando por la biología molecular.

Bibliography of Feral Goats:

http://www.erin.gov.au/biblio/frlgoats.html

Bibliografía comentada sobre Capra hircus (Artiodactyla)

Bibliography of the Icterini:

gopher://fmppr.fmnh.org:70/11/.fmnh/.acad/.zoo/.brd/ icterid

Referencias sobre este grupo de aves

Crocodilian Phylogenetics Bibliography:

http://www.med.jhu.edu/FAE/meers/croc.bib.phylo.html

Referencias sobre las relaciones filogenéticas de los cocodriliformes actuales y fósiles.

Indice Gopher de bibliografia ictiologica:

gopher://muse.bio.cornell.edu:70/77/.indices/fishbib/bib

Proporciona casi 14.000 referencias sobre estadios embrionarios y juveniles de los peces.

Kangaroo bibliography:

gopher://155.187.10.12:70/7waissrc\%3A/library/

bibliography/bibliography.src

Bibliografía sobre canguros.

Mamíferos Brasileiros: Uma Coletânea Bibliográfica:

http://www.bdt.org.br/bdt/biodiversitas/referencias

Contiene casi 2.000 referencias, con énfasis en ecología, etología y distribución de los mamíferos brasileños.

Neodat project bibliographic records:

gopher://gopher.keil.ukans.edu/77/.indices/neodat.ref/ neoref

Referencias de descripciones originales de especies de peces tropicales de agua dulce.
Red-Cockaded Woodpecker Bibliographies:

gopher://dewey.lib.ncsu.edu:70/11/library/disciplines/ biology/woodpecker

Esta bibliografía contiene artículos de revistas, libros, informes del gobierno americano, tesis y conferencias publicados desde 1980 sobre estos pícidos. Search the GooseRef Bibliography:

http://mendel.mbb.sfu.ca/gooseref/

Gestor simple para una base de datos con más de 1.700 registros de referencias científicas sobre la biología de varias especies de gansos. Se actualiza mensualmente.

B) Otras bases de datos online

Bibliography of Biodiversity Assessment Methodologies:

http://www.erin.gov.au/life/general_info/biodiv_assess_ intro.html

Referencias sobre metodologías de evaluación de la biodiversidad. Permite obtener un listado o realizar búsquedas selectivas.

Bibliographies-Internet links:

http://ice.ucdavis.edu/Cyberspace_Jump_Station/ bibliographies/

Listado de enlaces a bases de datos bibliográficas sobre diferentes temas relacionados con la ciencia y que están disponibles online .

\section{Cladistic Literature:}

http://www.utexas.edu/ftp/depts/systbiol/info/ cladliterature.html

Listado de referencias sobre cladismo.

Classification Literature Automated Search

Service: http://www.pitt.edu/ csna/class.html

Contiene referencias de revistas relacionadas con la clasificación.

Earth's Environmental Experts (3e)Ltd:

http://www.nhbs.co.uk/3e/

La 3E Database contiene información sobre expertos y especialistas en diferentes áreas de las ciencias ambientales.

History of Systematics:

http://rjohara.uncg.edu/files/biblio.histsyst

Publicaciones sobre la historia de la sistemática.

Introductory Systematics Bibliography:

http://rjohara.uncg.edu/files/biblio.clades

Bibliografía relacionada con los principios y la importancia de la sitemática contemporánea.

IUCN Publications Bibliography:

http://scilib.ucsd.edu/sio/indexes/iucn.html

Publicaciones de la International Union for Conservation of Nature \& Natural Resources desde 1948-1995. Información sobre especies en peligro y Biodiversidad. 
Oceanography on the Net. Library Resources \& Databases:

http://scilib.ucsd.edu/sio/guide/library.html\#BIOBIB

Conjunto de bases de datos bibliográficas dedicadas a temas relacionados con el mundo marino. Algunas de ellas han sido citadas en el apartado anterior.

Sequence Analysis and Comparison: A Bibliography: $h t t p: / / w w w . p i t t . e d u / \sim c s n a / d a y . h t m l$

Contiene 1.760 referencias sobre teoría y metodología de análisis de secuencias, alineamiento y comparación de secuencias, consensus, estima de filogenias a partir de secuencias y comparación o predicción de estructuras de secuencias.

$\underline{\text { Revistas }} \underline{\text { con servicio online }}$

En este apartado hemos incluido una relación de revistas de Zoología con servicio online. Aquellas revistas marcadas con un asterisco $(*)$ aparecen listadas en la categoría de Zoología en el Science Citation Index (1996).

A) Listado de revistas de Zoología

Abstracts of Entomology:

http://www.biosis.org/htmls/common/ento.html

Acarina:

http://members.aol.com/jccoke/society.html\#CIDA

Acta Theriologica (*):

http://bison.zbs.bialowieza.pl/acta/acta.html

Acta Tropica (*):

http://www.Elsevier.nl/inca/publications/store/5/0/6/0/4/

3/506043.pub.shtml

Acta Zoologica Sinica:

http://panda.ioz.ac.cn/ioz/zool.html

Acta Zoologica:

http://www.Elsevier.nl/inca/publications/store/6/5/5/ 655.pub.shtml

African Entomology (*):

http://www.up.ac.zalacademic/entomologicalsociety/journal.html

African Journal of Ecology (*):

http://www.blackwell-science.com/products journals/afje.htm

Alces Journal, (Moose):

http://www.lakeheadu.ca/ alceswww/journal.html

American Cetacean Society:

http://www.acsonline.org/ww.htm

American Entomologist:

http://www.entsoc.org/ae.htm

American Journal of Human Biology (*):

http://journals.wiley.com/wilcat-bin/ops/

ID0634051/1042-0533/prod
American Journal of Physical Anthropology (*): http://journals.wiley.com/wilcat-bin/ops/ ID0634051/0002-9483/prod

American Journal of Primatology (*):

http://journals.wiley.com/wilcat-bin/ops/ ID0634051/0275-2565/prod

American Naturalist (*):

http://www.journals.uchicago.edu/AN/home.html

American Zoologist (*):

http://www.sicb.org/public/publications/AZ/

Amphibian \& Reptile Conservation (ARC):

http://www.byu.edu/ arcon/

Anatomy and Embryology (*):

http://link.springer.de/link/service/journals/00429/index .htm

Animal Behaviour (*):

http://www.hbuk.co.uk/ap/journals/ar/

Animal Genetics (*):

http://www.blackwell-science.com/products/journals/ agen.htm

Annals of the Entomological Society of America (*): http://www.entsoc.org/annals.htm

Année Biologique, L' (*):

http://www.gauthier-villars. fr/

Annual Review of Ecology and Systematics (*):

http://www.annurev.org/series/ecol\&sys/ecol\&sys.htm

Annual Review of Entomology (*):

http://www.annurev.org/series/entomol/entomol.htm

Annual Review of Fish Diseases (*):

http://www.elsevier.nl/inca/publications/store/9/6/3/963. pub.shtml

Antarctic Science (*):

http://www.blackwell-science.com/products/journals/ antar.htm

Apidologie (*):

http://www.elsevier.nl/inca/publications/store/5/0/5/7/9/ 6/505796.pub.shtml

Applied Animal Behaviour Science (*):

http://www.Elsevier.nl/inca/publications/store/5/0/3/3/0/

1/503301.pub.shtml

Aquaculture Economics and Management:

http://www.blackwell-science.com/products/journals/ aem.htm

Aquaculture International:

http://www.chapmanhall.com/aq/default.html

Aquaculture Nutrition:

http://www.blackwell-science.com/products/journals/ anut.htm

Aquaculture Research:

http://www.blackwell-science.com/products/journals/ ares.htm

Aquarium Science and Conservation:

http://www.chapmanhall.com/as/default.html 
Aquatic Conservation:

http://www.chapmanhall.com/as/default.html

Aquatic Ecology:

http://www.wkap.nl/kapis/CGI-BIN/WORLD/ journalhome.htm? 1386-2588

Aquatic Microbial Ecology (*):

http://www.bio.net:80/bioarchives/BIO-JOURNALS/

AQUATIC_MICROBIAL_ECOLOGY/

Archives of Insect Biochemistry and Physiology:

http://journals.wiley.com/wilcat-bin/ops/ ID0634051/0739-4462/prod

Argia: http://www.afn.org/ iori/dsapubls.html

Atualidades Ornitologicas:

http://www.ao.com.br/

Auk, The: A Quarterly Journal of Ornithology (*): http://www.nmnh.si.edu/BIRDNET/AOU/auklet.html

Australasian Shell News:

http://www.austmus.gov.au/htm/mal/malsoc/newslet1.htm

Australian Journal of Ecology (*):

http://www.blackwell-science.com/products/journals/ xaje.htm

Australian Journal of Entomology (*):

http://www.uq.edu.au/entomology/aes/journal.html

Australian Journal of Zoology (*):

http://www.publish.csiro.au/journals

Australian Mammalogy:

http://ikarus.jcu.edu.au/mammal/aminfo.html

Avian Pathology (*): http://www.bdt.org.br/bioline/ap

Behavioral Ecology and Sociobiology (*):

gopher://trick.ntp.springer.de/11/TOC/265/

Behavioral Ecology (*):

http://www.oup.co.uk/jnls/list/beheco/

Beiträge zur Entomologie:

http://journals.wiley.com/wilcat-bin/ops/

ID0634051/0005-805X/prod

Biochemical Systematics and Ecology (*):

http://www.Elsevier.nl/inca/publications/store/3/6/4/364.

pub.shtml

Biodiversity and Conservation (*):

http://www.chapmanhall.com/bc/default.html

Biodiversity Letters:

http://www.chapmanhall.com/bl/default.html

Biological Bulletin, The $(*)$ :

http://tbone.biol.scarolina.edu/ dean/carl/The-

Biological-bulletin.html

Biological Conservation (*):

http://www.elsevier.nl/inca/publications/store/4/0/5/8/5/

3/405853.pub.shtml

Biological Journal of the Linnean Society (*):

http://www.hbuk.co.uk/ap/journals/bj.htm

Biological Reviews of the Cambridge Philosophical Society:

http://www.cup.cam.ac.uk/journals/jnlscat/bre/bre.html
Biometrical Journal (*):

http://journals.wiley.com/wilcat-bin/ops/ ID0634051/0323-3847/prod

Biometrika (*): http://www.oup.co.uk/jnls/list/biomet/ BioScience Magazine (*):

http://www2.aibs.org/aibs/bioscience.html

Biotropica (*):

http://atb.botany.ufl.edu/atb/biotropica/biotropica.html

Bird Conservation International:

http://www.cup.cam.ac.uk/journals/jnlscat/bci/bci.html

BirdScope:

http://www.ornith.cornell.edu/Pubs/BS/main.html

Bulletin of American Odonatology:

http://www.afn.org/ iori/dsapubls.html

Bulletin of the Ecological Society of America:

http://www.sdsc.edu/projects/ESA/esapubs.htm\#PubsB. html

Bulletin of the Malacological Society of London: http://www.sunderland.ac.uk/ esOmda/jms.htm\#Bulletin

Bulletin of Marine Science (*):

http://www.rsmas.miami.edu/bms/

Bulletin of Zoological Nomenclature:

http://www.iczn.org/

Canadian Journal of Fisheries and Aquatic Sciences (*):

http://www.nrc.ca/cisti/journals/cjfas.html

Canadian Journal of Zoology (*):

http://www.cisti.nrc.ca/cisti/journals/rjzoo.html

Chromosome Research:

http://www.chapmanhall.com/ch/default.html

Cladistics (*):

http://www.vims.edu/ mes/hennig/journal.html

Coleopterists Bulletin (*):

http://www.auburn.edu/beetles/colebull.htm

Computer Applications in the Biosciences (*):

http://www.oup.co.uk/jnls/list/cabios/

Condor (*):

http://www.nmnh.si.edu/BIRDNET/COS/authors.html

Conservation Biology (*):

http://www.blackwell-science.com/products/journals/ xconb.htm

Conservation Ecology:

http://www.sdsc.edu/ESA/esapubs.htm\#PubsA.html

Continental Shelf Research $(*)$ :

http://www.elsevier.nl/inca/publications/store/6/6/2/

Copeia (*):

http://www.utexas.edu/depts/asih/pubs/copeiapr.html

Coral Reefs (*):

gopher://trick.ntp.springer.de/11/TOC/338/

Current Advances in Ecological \& Environmental

Sciences:

http://www.elsevier.nl:80/inca/publications/store/4/6/7/4 67.pub.shtml 
Cybium: http://www.mnhn.fr/sfi/sfi.html\#Cybium

Deep Sea Research Part I: Oceanographic Research Papers (*):

http://www.elsevier.nl/inca/publications/store/2/1/6/216. pub.shtml

Deep Sea Research Part II: Topical Studies in Oceanography (*):

http://www.elsevier.nl/inca/publications/store/1/1/6/116. pub.shtml

Deutsche Entomologische Zeitschrift (*):

http://journals.wiley.com/wilcat-bin/ops/ ID0634051/0012-0073/prod

Developmental Biology (*):

http://www.apnet.com/www/journal/db.htm

Diseases of Aquatic Organisms (*):

http://www.int-res.com/int-res/dao/dao.html

Diversity and Distributions:

http://www.blackwell-science.com/products/journals/ biod.htm

Dolphin Project Europe:

http://www.envirolink.org/archives/enews/0020.html

Earth Island Journal:

http://www.earthisland.org/ei/journal/journal.html

Ecography (*):

http://www.oikos.ekol.lu.se/Ecography.jrnl.html

Ecological Abstracts:

http://www.elsevier.nl:80/inca/publications/store/4/0/5/8

/5/5/

Ecological Applications (*):

http://www.sdsc.edu/ESA/esapubs.htm\#PubsA.html

Ecological Bulletins:

http://www.oikos.ekol.lu.se/EB.jrnl.html

Ecological Engineering (*):

http://www.elsevier.nl/inca/publications/store/5/2/2/7/5/

1/522751.pub.shtml

Ecological Entomology (*):

http://www.blackwell-science.com/products/journals/ eent.htm

Ecological Modelling (*):

http://www.elsevier.nl/locate/ecolmodel

Ecological Monographs (*):

http://www.sdsc.edu/ESA/esapubs.htm\#PubsA.html $\mathbf{y}$

gopher://agate.lut.ac.uk:4077/11/Ecological\%20

Monographs

Ecological Research (*):

http://www.blackwell-science.com/products/journals/ xeres.htm

Ecology \& Systematics:

http://www.annurev.org/series/ecol\&sys/ecol\&sys.htm

Ecology (*):

http://www.sdsc.edu/ESA/esapubs.htm\#PubsA.html

Ecology Letters:

http://www.blackwell-science.com/products/journals/ele.htm
EcoNews Africa:

http://www.web.apc.org/ econews/

Electrophoresis:

http://journals.wiley.com/wilcat-bin/ops/ ID0634051/0173-0835/prod

Emu (*):

http://www.vicnet.net.au/ raou/emu/emu.html

Entomologia Experimentalis et Applicata:

http://www.wkap.nl/kapis/CGI-BIN/WORLD/

journalhome.htm?0013-8703

Entomologia Sinica:

http://panda.ioz.ac.cn/ioz/es0.html

Entomologica Scandinavica (*):

http://www.aki.ku.dk/zmuc/ento/ent_sca.htm

Environmental and Ecological Statistics:

http://www.chapmanhall.com/es/default.html

Environmental Biology of Fishes (*):

http://www.wkap.nl/kapis/CGI-BIN/WORLD/

journalhome.htm?0378-1909

Environmental Entomology (*):

http://www.entsoc.org/ee.htm

Environmental Monitoring and Assessment (*):

http://www.wkap.nl/kapis/CGI-BIN/WORLD/

journalhome.htm?0167-6369

Environmentalist, The:

http://www.chapmanhall.com/en/default.html

Estuarine, Coastal and Shelf Science (*):

http://www.hbuk.co.uk/ap/journals/ec.htm

Ethology (*):

http://www.blackwell-science.com/products/journals/ xeth.htm

European Journal of Entomology (*):

http://www.jcu.cz/ entu/eje.htm

Evolution (*):

ht tp://www.bio.net: $80 /$ bioarchives/ BIO-JOURNALS/EVOLUTION/

Evolutionary Anthropology:

http://journals.wiley.com/wilcat-bin/ops/ ID0634051/1060-1538/prod

Evolutionary Computation:

http://www-mitpress.mit.edu/jrnls-catalog/ evolution.html

Evolutionary Ecology $(*)$ :

http://www.chapmanhall.com/ee/default.html

Exotic Birds:

http://kaos.erin.gov.au/life/species/fauna/exotic_birds/ exotic_birds.html

Experimental and Applied Acarology (*):

http://www.chapmanhall.com/ea/default.html

Experimental Biology Online:

http://science.springer.de/ebo/ebo-main.htm

Fish and Fisheries Research:

http://WWW.LSOFT.COM/FFResearch/ 
Fisheries Management and Ecology:

http://www.blackwell-science.com/products/journals/ fme.htm

Fisheries Oceanography:

http://www.blackwell-science.com/products/journals/ fog.htm

Fisheries Research (*):

http://www.elsevier.nl/inca/publications/store/5/0/3/3/0/ 9/503309.pub.shtml

Flightlines:

http://kaos.erin.gov.au/life/species/fauna/flightlines/ flightlines.html

Florida Entomologist:

http://www.fcla.ufl.edu/FlaEnt/fehmpg.htm

Folia Biologica (*):

http://www.apnet.com/www/catalog/fb.htm

Folia Malacologica:

http://hum.amu.edu.pl/ polmal/folia/folia.htm

Folia Primatologica (*):

http://www.karger.ch/journals/fpr/fprdes.htm

Forest Ecology and Management (*):

http://www.elsevier.nl/locate/foreco

Freshwater Biology (*):

http://www.blackwell-science.com/products/journals/ fwb.htm

Functional Ecology (*):

http://www.blackwell-science.com/products/journals/ fecol.htm

Genetics, Selection, Evolution (*):

http://www.Elsevier.nl/inca/publications/store/5/0/5/7/9/ 7/505797.pub.shtml

Genome (*):

http://www.cisti.nrc.ca:80/cisti/journals/tocgen.html

Genome Research (*):

http://www.cshl.org/journals/gr/

Genomics (*):

http://www.apnet.com/www/journal/ge.htm

Global Change Biology (*):

http://www.blackwell-science.com/products/journals/ gcb.htm

Global Ecology and Biogeography Letters (*):

http://www.blackwell-science.com/products/journals/ gebl.htm

Grass and Forage Science (*):

http://www.blackwell-science.com/products/journals/ gfs.htm

Hawaiian Shell News:

http://www.hits.net/ hsn/

Helminthological Abstracts:

http://WWW.CABI.ORG/catalog/journals/absjour/0h.htm Heredity (*):

http://www.blackwell-science.com/products/journals/ hered.htm
Herpetologica $(*)$ :

http://130.160.104.76/HL/Titles.html

Holartic Lepidoptera:

http://www.troplep.org/hl.htm

Hydrobiologia (*):

http://www.wkap.nl/kapis/CGI-BIN/WORLD/journalhome. htm?0018-8158

ICES - Journal of Marine Science (*):

http://www.hbuk.co.uk/ap/journals/jm.htm

Insect Biochemistry and Molecular Biology (*):

http://www.elsevier.nl:80/inca/publications/store/3/9/0/3

90.pub.shtml

Insect Molecular Biology:

http://www.blackwell-science.com/products/journals/ imb.htm

International Journal for Parasitology (*):

http://www.elsevier.nl:80/inca/publications/store/3/5/3/3

53.pub.shtml

International Journal of Insect Morphology and Embryology (*):

http://www.Elsevier.nl/inca/publications/store/3/5/6/356 .pub.shtml

International Journal of Sustainable Development and World Ecology, The:

http://www.parthpub.com/susdev/home.html

Internationale Revue der Gesamten Hydrobiologie (*):

http://journals.wiley.com/wilcat-bin/ops/ ID0634051/0020-9309/prod

Invertebrate Taxonomy:

http://www.publish.csiro.au/journals/it/index.html

Journal of African Zoology:

http://www.biol.ucl.ac.belecol/JAZ/JAZ.HomePage.html Journal of Anatomy (*):

http://www.cup.cam.ac.uk/journals/jnlscat/ana/ana.html Journal of Animal Breeding and Genetics (*):

http://www.blackwell-science.com/products/journals/ xjabg.htm

Journal of Animal Ecology (*):

http://www.blackwell-science.com/products/journals/ janim.htm

Journal of Animal Physiology and Animal Nutrition (*):

http://www.blackwell-science.com/products/journals/ xjapn.htm

Journal of Apicultural Research:

http://www.cardiff.ac.uk/ibra/jar.html

Journal of Applied Ecology (*):

http://www.blackwell-science.com/products/journals/ jappl.htm

Journal of Applied Entomology (*):

http://www.blackwell-science.com/products/journals/ xjae.htm 
Journal of Applied Ichthyology (*):

http://www.blackwell-science.com/products/journals/ xjai.htm

Journal of Aquatic Ecosystem Stress and Recovery: http://www.wkap.nl/kapis/CGI-BIN/WORLD/journalhome. htm?1386-1980

Journal of Arid Environments (*):

http://www.hbuk.co.uk/ap/journals/ael

Journal of Avian Biology (*):

http://www.oikos.ekol.lu.se/JAB.jrnl.html

Journal of Biogeography (*):

http://www.blackwell-science.com/products/journals/ jbiog.htm

Journal of Classification (*):

http://www.pitt.edu/ csna/joc.html

Journal of Coastal Conservation:

http://www2.passagen.se/opulus.com/JCC/jcchome.html

Journal of Comparative Physiology B:

Biochemical, Systemic, and Environmental Physiology (*):

http://www.springer-ny.com/catalog/np/biomed/ 0174-1578.html

Journal of Comparative Psychology (*):

http://www.apa.org/journals/com.html

Journal of Crustacean Biology, The (*):

http://www.lam.mus.ca.us/ tcs/jcb.html

Journal of Ecology (*):

http://www.blackwell-science.com/products/journals/ jecol.htm

Journal of Economic Entomology (*):

http://www.entsoc.org/jee.htm

Journal of Ethology (*):

http://neco.biology.kyushu-u.ac.jp/ eiiti/JE/JEinst.html

Journal of Evolutionary Biology (*):

http://www.unibas.ch/dib/eseb/esebhome.htm

Journal of Experimental Marine Biology and Ecology (*):

http://www.elsevier.nl/inca/publications/store/5/2/3/0/1/1/

Journal of Experimental Zoology, The (*):

http://journals.wiley.com/wilcat-bin/ops/ ID0634051/0022-104X/prod

Journal of Field Ornithology (*):

http://www.nmnh.si.edu/BIRDNET/AFO/journal.html

Journal of Fish Biology (*):

http://www.hbuk.co.uk/ap/journals/jb.htm

Journal of Fish Diseases (*):

http://www.blackwell-science.com/products/journals/ jfd.htm

Journal of Great Lakes Research (*):

http://www.geog.buffalo.edu/GLP/iaglr/jglr/index.html

Journal of Helminthology (*):

http://WWW.CABI.ORG/catalog/journals/primjour/

jouhelm.htm
Journal of Herpetology (*):

http://falcon.cc.ukans.edu/ gpisani/JHprev.html

Journal of Human Evolution (*):

http://www.hbuk.co.uk/ap/journals/hu/

Journal of Insect Conservation:

http://www.chapmanhall.com/is/default.html

Journal of Insect Physiology (*):

http://www.elsevier.nl:80/inca/publications/store/2/3/1/

Journal of Invertebrate Pathology (*):

http://www.apnet.com/www/journal/in.htm

Journal of Mammalogy (*):

http://wkuweb1.wku.edu/ asm/journal.html

Journal of Molecular Biology (*):

http://www.hbuk.co.uk/ap/journals/mb/

Journal of Molluscan Studies, The (*):

http://www.oup.co.uk/jnls/list/mollus/

Journal of Morphology (*):

http://journals.wiley.com/wilcat-bin/ops/ ID0634051/0362-2525/prod

Journal of Natural History (*):

http://www.tandf.co.uk/jnls/nah.htm

Journal of Oceanography:

http://www.wkap.nl/kapis/CGI-BIN/WORLD/journalhome. htm?0916-8370

Journal of Oregon Ornithology:

http://www.orednet.org/ rbayer/j/joomenu.htm

Journal of Orthoptera Research:

http://www.uwyo.edu/ag/psisci/lockwood/jor4.htm

Journal of Plankton Research, The (*):

http://www.oup.co.uk/jnls/list/plankt/

Journal of the International Goat Association:

http://www.elsevier.nl/inca/publications/store/5/0/3/3/1/

7/503317.pub.shtml

Journal of the Marine Biological Association of the United Kingdom $\left({ }^{*}\right)$ :

http://www.cup.cam.ac.uk/journals/jnlscat/mbi/mbi.html Journal of Marine Systems (*):

http://www.elsevier.nl/inca/publications/store/5/ $0 / 3 / 3 / 4 / 4 /$

Journal of the North American Benthological Society, The:

http://www.benthos.org/jnabs

Journal of the Royal AustralAsian Ornithologist

Union - Emu:

http://avoca.vicnet.net.au/ birdsaus/emu/emu.html

Journal of the Royal Society of New Zealand (*):

http://www.rsnz.govt.nz/www/sir/journals/royal/royal.html

Journal of the Ukranian Entomological Society:

http://www.york.biosis.org/zrdocs/zoolinfo/j_ukrent.htm

Journal of Theoretical Biology (*):

http://www.hbuk.co.uk/ap/journals/jt.htm

Journal of Tropical Ecology (*):

http://www.cup.cam.ac.uk/journals/jnlscat/tro/tro.html 
Journal of Zoological Systematics and Evolutionary Research (*):

http://www.blackwell-science.com/products/journals/ xjzer.htm

Journal of Zoology (*):

http://www.oup.co.uk/jnls/list/zoo/

Korean Journal of Parasitology:

http://sun.hallym.ac.kr/ shuh/kjp.html

Lakes and Reservoirs: Research and Management:

http://www.blackwell-science.com/products/journals/ xlare.htm

Limnology and Oceanography (*): http://aslo.org/lo/ Litteratura Serpentium:

http://pcm0053.let.ruu.nl/ess/litt_eng.htm

Loon: http://biosci.cbs.umn.edu/ mou/loon/

Malacologia (*):

http://www.ucmp.berkeley.edu/mologis/Malcogia.html

Mammal Review (*):

http://www.blackwell-science.com/products/journals/ mam.htm

Mammalian Genome (*):

http://link.springer.de/link/service/journals/00335/ index.htm

Marine \& Freshwater Research (*):

http://www.publish.csiro.au/journals/mfr/index.html

Marine Biology (*):

http://link.springer.de/link/service/journals/00227/ index.htm

Marine Ecology:

http://www.blackwell-science.com/products/journals/ xmeco.htm

Marine Ecology Progress Series (*):

http://www.int-res.com/int-res/meps/meps.html

Marine Environmental Research (*):

http://www.Elsevier.nl/inca/publications/store/4/0/5/8/6/

5/405865.pub.shtml

Marine Mammals Science (*):

http://pegasus.cc.ucf.edu/ smm/mms.htm

Medical and Veterinary Entomology (*):

http://www.blacksci.co.uk/products/journals/mve.htm

Microfauna Marina:

http://www.publish.csiro.au/journals/wr/index.html

Mitteilungen aus dem Zoologischen Museum in Berlin:

http://journals.wiley.com/wilcat-bin/ops/ ID0634051/0373-8493/prod

Molecular and Biochemical Parasitology (*):

http://www.elsevier.nl/locate/molbiopara

Molecular Biology and Evolution (*):

http://www.hgc.sph.uth.tmc.edu/smbe

Molecular Ecology (*):

http://www.blackwell-science.com/products/journals/ mecol.htm
Molecular Marine Biology and Biotechnology (*): http://www.blackwell-science.com/products/journals/ xmmbb.htm

Molecular Phylogenetics and Evolution (*):

http://www.apnet.com/www/journal/fy.htm

Molluscan Research:

http://www.austmus.gov.au/htm/mal/malsoc/journ 1.htm

Natural Areas Journal (*):

http://www.vmedia.com/naj/

Nature (*): http://www.nature.com/

Naturwissenschaften $(*)$ :

http://link.springer.de/link/service/journals/00114/index.

htm

New Environmentalist, The:

gopher://cais.cais.com:70/11/.manning

New Zealand Journal of Marine and Freshwater Research (*):

http://www.rsnz.govt.nz/www/sir/journals/marine/ marine.html

New Zealand Journal of Zoology (*):

http://www.rsnz.govt.nz/www/sir/journals/zoology lzoology.html

North American Journal of Fisheries Management:

http://www.esd.ornl.gov/societies/AFS/najfm.html

Nova Supplementa Entomologica:

http://www.biologie.uni-ulm.de/extern/dei-eberswaldel dei-nova.html

Oceanographic Literature Review:

http://www.vicnet.net.au/ iffa/sample.htm

Oceanography Package:

http://www.Elsevier.nl/inca/publications/store/6/0/0/1/3/ 3/600133.pub.shtml

Oceanus (*):

http://www.whoi.edu/oceanus/

Odonatologica:

http://www2.arnes.si/ mbsodonad1/18e.html

Oecologia (*):

http://link.springer.de/link/service/journals/00442/ index.htm

Oikos (*): http://oikos.ekol.lu.se/Oikosjrnl.html

Ophelia (*): http://inet.uni-c.dk/ marilab/ophelia.htm Oryx:

http://www.blackwell-science.com/products/journals/ oryx.htm

Parasite Inmunology (*):

http://www.blackwell-science.com/products/journals/ pimm.htm

Parasite Immunology (*):

http://www.blacksci.co.uk/products/journals/pimm.htm

Parasitology International:

http://www.Elsevier.nl/inca/publications/store/6/0/0/1/1/

1/600111.pub.shtml 
Parasitology Today (*):

http://www.elsevier.nl:80/inca/publications/store/4/0/5/9 /1/5/405915.pub.shtml

Parasitology (*):

http://www.cup.cam.ac.uk/journals/jnlscat/par/par.html

Philosophical Transactions: Biological Sciences: http://www.pubs.royalsoc.ac.uk/publish/phi_bs/index.htm Physiological Entomology:

http://www.blackwell-science.com/

Polar Biology (*):

http://link.springer.de/link/service/journals/00300/ index.htm

Physiology and Behavior:

http://www.elsevier.nl/locate/physbeh

Proceeding Biological Sciences:

http://www.pubs.royalsoc.ac.uk/publish/pro bs/index.htm

Proceedings of the National Academy of Sciences

USA (*): http://www.pnas.org/

Progress in Oceanography $(*)$ :

http://www.Elsevier.nl/inca/publications/store/4/2/2/

422.pub.shtml

Records of the Australian Museum:

http://www.austmus.gov.au/

Researches on Population Ecology (*):

http://mcc515.ed.shizuoka.ac.jp/POPECOL/RP.html

Restoration Ecology:

http://www.blackwell-science.com/products/journals/

xreco.htm

Reviews in Fish Biology and Fisheries (*):

http://www.chapmanhall.com/rf/default.html

Russian Journal of Herpetology:

http://www.folium.ru/rjh/

Russian Journal of Nematology:

http://www.scri.sari.ac.uk/rjn/rjn.htm

Sarsia (*): http://www.ifm.uib.no/sarsia/last_no.htm

Science (*): http://www.sciencemag.org/

Scientific American (*): http://www.sciam.com/

Scientist, The (*):

http://ds.internic.net/pub/the-scientist/

Shell, The (La Conchiglia):

http://www.conch.pronet.it/

Small Ruminant Research (*):

http://www.elsevier.nl/inca/publications/store/5/0/3/3/1/

7/503317.pub.shtml

Spirula: http://home.pi.net/ spirula/

Systematic Biology (*):

http://www.utexas.edu/depts/systbiol/

Systematic Entomology (*):

http://www.blackwell-science.com/products/journals/ sent.htm

Systematic Parasitology (*):

http://www.wkap.nl/kapis/CGI-BIN/WORLD/journalhome. htm?0165-5752
Theoretical Population Biology (*):

http://www.apnet.com/www/journal/tp.htm

Today's Aquarist:

http://www.cadvision.com/nolimits/Todays_Aquarist.html Tragopan:

http://www.open.ac.uk/OU/Academic/Biology/P_McGowal TRAGOPAN/TRAGOPAN_3.html

Transactions of the American Fisheries Society: http://www.esd.ornl.gov/societies/AFS/trans.html

Trends in Biological Science:

http://www.elsevier.nl/locate/tibs

Trends in Ecology and Evolution (*):

http://www.elsevier.nl/locate/tree

Trends in Genetics (*):

http://www.elsevier.nl/locate/tig

Tropical Biodiversity:

http://www.bdt.org.br/bioline/tb

Tropical Lepidoptera:

http://www.troplep.org/tl.htm

Tryonia:

gopher://erato.acnatsci.org:70/11/.publications

Veliger, The (*):

http://www.ucmp.berkeley.edu/mologis/Veliger.html

Vita Marina (Moluscos):

http://home.pi.net/ spirula/

Vivarium Magazine:

http://www.thevivarium.com/vivmag/MagIndx.html

Water, Air, and Soil Pollution (*):

http://www.wkap.nl/kapis/CGI-BIN/WORLD/journalhome. htm?0049-6979

Wetlands - The SWS Quartery Journal (*):

http://www.sws.org/wetlands.html

Wetlands Ecology and Management:

http://www.wkap.nl/kapis/CGI-BIN/WORLD/journalhome. htm?0923-4861

Whales Alive:

http://elfnetla.elfi.com/csihome.html (newsletter)

Whalewatcher Journal:

http://www.acsonline.org/ww.htm

Wildlife Research (*):

http://www.publish.csiro.au/journals/wr/index.html

Wilson Bulletin, The (*) (ornitología):

http://www.ummz.lsa.umich.edu/birds/bulletin.html

Zoo Biology (*):

http://journals.wiley.com/wilcat-bin/ops/ ID0634051/0733-3188/prod

Zoologica Scripta (*):

http://www.elsevier.nl/inca/publications/store/6/7/0/670. pub.shtml

Zoological Journal of the Linnean Society (*):

http://www.hbuk.co.uk/ap/journals/zj/

Zoology:

http://www.publish.csiro.au/journals/ajz/index.html 


\section{Zoomorphology:}

http://link.springer.de/link/service/journals/00435/ index.htm

B) Servidores que mantienen listados de revistas

Biological Journals and Abbreviations:

http://arachne.prl.msu.edu/journams/

Directorio de revistas de biología y medicina, con sus abreviaturas. No todas están en Internet.

Ecological Journals:

http://www.ng.hik.se/ nmato/journals.html

Una selección de revistas de biología y ecología dirigida especialmente a la biología marina.

Journaux Browse (Life Sciences) gopher in France: gopher://gopher.genethon.fr/11/Biblio/Journaux

Catálogo de revistas de biología general.

Journal List. Auburn University Librairies:

http://www.lib.auburn.edu/scitech/docs/Journallist.html

Listado de revistas de ciencia y tecnología.

\section{Molluscan Journals:}

http://www.ucmp.berkeley.edu/mologis/AutInst.html

Ofrece un listado de revistas sobre malacología

con las instrucciones de publicación de cada una de ellas.

\section{Phylogenetics Publications:}

http://www.ucmp.berkeley.edu/subway/phylo/phylopub.html

Listado de revistas, newsletters y otras publicaciones relacionadas con Sistemática y Taxonomía.

\section{Libros y Documentos Online}

Estos son algunos ejemplos de edición electrónica de libros en el área que nos ocupa, si bien comienzan a ser frecuentes este tipo de iniciativas en muchas áreas (visitar http://www.edoc.com/jrlbin/wilma/opp, para ejemplos de proyectos editoriales online)

Systematics Agenda 2000:

gopher://muse.bio.cornell.edu:70/11/standards/sa2000

Declaración de objetivos de biología sistemática que documenta la importancia del trabajo de estos científicos.

The Origin of Species y Voyage of the Beagle by Charles Darwin:

http://www.literature.org/Works/Charles-Darwin/

Rhythms in organisms - An introduction for observing, experimenting, recording and analysing:

http://bioclox.bot.biologie.uni-tuebingen.de/ Html we/Buch/ren96/ren96.html

Biodiversidad de América Latina:

http://dell.ieco.conacyt.mx/bd/bdconten.htm

Este libro es el primero en una serie sobre la biodiversidad de los países latinoamericanos.
World Conservation Monitoring Centre:

Publications:

http://www.wcmc.org.uk:80/information_services/ publications/

Los libros que se citan a continuación se encuentran online, aunque también pueden adquirirse editados en papel (http://www.iucn.org/bookstore/index.html):

WCMC Biodiversity Series: Biodiversity Data Sourcebook, The Biodiversity Clearing House Concept And Challenges, Priorities for Conserving Global Species Richness and Endemism, The Diversity of the Seas: A regional approach, Assessing Biodiversity Status and Sustainability.

WCMC Biodiversity Bulletin: Priorities for Biodiversity Conservation in the Tropics Other Publications: Guide to Information Management y Private Protected Areas.

Publicaciones electrónicas

A) Revistas electrónicas

Directory of Electronical Journals and Newsletters: http://arl.cni.org/scomm/edir/index.html

Es un directorio de contenido general.

E.journal: http://www.edoc.com/ejournal/

Se trata de una base de datos de revistas electrónicas donde las busquedas pueden hacerse por categorías temáticas y palabras clave.

B) CD-Rom

Biological and Agricultural Index:

http://www.hwwilson.com/bai.htm

Publicado por H.W. Wilson. Es una base de datos que cubre 266 publicaciones periódicas de biología y agricultura. También se puede consultar online.

The International Center for Living Aquatic Resources Management (ICLARM):

http://www.cgiar.org/iclarm/pubsof/icpub1.htm

Organización internacional de investigación dedicada a la mejora de la productividad y manejo de recursos acuáticos, publica bases de datos en CD-Rom sobre todos los aspectos de la ictiología (FishBase 96) y los recursos de los arrecifes de coral (ReefBase).

Multimedia Encyclopedia of Mammalian Biology:

http://www.mcgraw-hill.com.au/professional/ m_comingsoon.html

Incluye los cinco volúmenes de la Grzimek's Encyclopedia of Mammals, además cuenta con secuencias de vídeo y audio que fueron proporcionadas por la BBC Natural History Archives.

NISC: http://www.nisc.com/

Estas bases de datos bibliográficas cubren un amplio rango de temas de ciencias naturales y 
sociales, artes y humanidades. Aquatic Sciences and Fisheries Abstracts (ASFA), Aquatic Biology, Aquaculture \& Fisheries Resources, Arctic \& Antarctic Regions, Biotechnology Abstracts: Agricultural \& Environmental, Ecology Abstracts, Entomology Abstracts, Environmental Periodicals Bibliography, Essential Ecology, Zoology \& Plant Science Abstracts, Essential Fisheries Abstracts, Essential Forestry \& Wildfire Abstracts, Essential Ornithological Abstracts, Essential Wildlife \& Conservation Biology Abstracts, Fish \& Fisheries Worldwide, Marine, Oceanographic \& Freshwater Resources, Species Information Library, Water Resources Abstracts, Water Resources Worldwide y Wildlife Worldwide.

\section{Root-Knot Nematode Taxonomic Database:}

http://www.cabi.org/catalog/cdrom/rootknot.htm

Este CD contiene referencias y muchos recursos sobre este grupo de nematodos.

\section{SilverPlatter Databases:}

http://www.silverplatter.com/catalog.htm

SilverPlatter proporciona acceso electrónico a bases de datos bibliográficas o textos. Cuenta con un amplio catálogo de bases de datos en CD-ROM sobre temas relacionados con Zoología: Aquatic Sciences and Fisheries Abstracts (ASFA), Biological Abstracts, Biological Abstracts/RRM (Reports, Reviews, Meetings), BIOSIS GenRef (genética), BIOSIS Previews on Compact Disc (información mensual de biología), EARTHDISC (geología, geografía y oceanografía), ECODISC (ecología y ecosistemas), Life Sciences Collection, The OCLC Environment Library (ciencias ambientales), Wilson Biological \& Agricultural Index y Zoological Record.

ETI:

http://wwweti.eti.bio.uva.nl/cdroms/default.shtml

Los CD-ROMs publicados en la actualidad son los siguientes: Birds of Europe, Bird Remains Identification System, Fishes of the North-Eastern Atlantic and the Mediterranean Five Kingdoms, Lobsters of the World, Marine Mammals of the World, Marine Planarians of the World, Pelagic Molluscs of the World, North Australian Sea Cucumbers.

Para el año 1997 preveen publicar los siguientes: Bats of India, Braconidae, Copepods. Aetidaeidae of the World, Lemurs of Madagascar and the Comorans, Marine, Brackish \& Freshwater Ostracods Of NW Europe, Turbellarian Families and Genera, Turtles of the World.

\section{GEOBASE CD-ROM:}

http://www.elsevier.nl:80/inca/publications/store/3/0/6/5 /8/30658.pub.shtml http://www.silverplatter.com/catalog/elge.htm

Publicado por Elsevier Science. Geobase es una base de datos bibliográfica sobre literatura científica en geografía física y humana, ciencias de la tierra, ciencias ambientales, ecología y disciplinas relacionadas.

\section{Organizaciones y Centros de Información}

\section{Organizaciones Mundiales}

\section{Biodiversity Information Network-BIN21:} http://www.bdt.org.br/bin21/bin21.html

Es una red sobre biodiversidad que pretende reunir la información relevante sobre este tema y hacerla asequible electrónicamente y por otros medios.

CAB International (CAB): http://www.cabi.org/

Es una organización intergubernamental que facilita información, publicaciones, y servicios científicos a nivel mundial sobre agricultura, silvicultura y manejo de recursos naturales. Mantiene la mayor base de datos del mundo sobre estos temas (CAB Abstracts). Está constituida por cuatro instituciones: International Institute of Biological Control, International Mycological Institute, International Institute of Parasitology e International Institute of Entomology.

Committee on Data for Science and Technology (CODATA):

http://www.lmcp.jussieu.fr/icsu/Structure/codata.html http://www.nrc.ca/programs/codata/

Comité científico del International Council of Scientific Unions (ICSU) (http://www.lmcp.jussieu.fr/icsu/). Trabaja sobre una base interdisciplinar y persigue mejorar la calidad, fiabilidad, proceso, manejo y accesibilidad de los datos científicos y tecnológicos.

\section{Convention on Biological Diversity:}

http://www.biodiv.org/

En este web puede encontrarse toda la información la historia y actualidad del Convenio de Diversidad Biológica.

\section{Conservation International (CI):}

http://www.conservation.org/

Organización que protege las áreas de riqueza biológica de la Tierra para ayudar a sus poblaciones humanas a mejorar su calidad de vida.

Global Action and Information Network:

http://www.igc.apc.org/gain/

Este servidor está diseñado para facilitar el acceso a la información sobre política y legislación ambiental y conservación del ambiente. 
Global Environment Facility (GEF):

http://www.worldbank.org/html/gef/gefgraph.htm

El GEF proporciona ayudas para proyectos y programas que promuevan la protección del medio ambiente global y su desarrollo económico sostenido. ICUN-The World Conservation Union: http://w3.iprolink.ch/iucnlib/

Tiene como misión proporcionar conocimiento y dirección para el uso adecuado de los recursos naturales. Publica manuales, guías, informes y documentos con directrices para la conservación de la biodiversidad.

International Centre for Living Aquatic Resources Management (ICLARM):

http://www.cgiar.org/ICLARM/

Organización científica internacional orientada a mejorar la productividad y manejo de los recursos acuáticos para el beneficio de usuarios y consumidores en paises en desarrollo.

International Institute for Applied Systems Analysis (IIASA): $h t t p: / / w w w . i i a s a . a c . a t /$

Organización científica no gubernamental que agrupa expertos de varias disciplinas cuyas actividades están orientadas a temas de medio ambiente, economía tecnología y sociedad, en el contexto de las dimensiones humanas del cambio global.

International Union for Biological Sciences (IUBS), Taxonomic Database Working Group: http://trident.ftc.nrcs.usda.gov/npdc/18tdwg.html

Hasta hace poco ha dirigido los estándares para las bases de datos taxonómicas de plantas. En la actualidad sus esfuerzos se dirigen también hacia la Zoología.

Species 2000: http://www.sp2000.org/

Especies 2000 tiene como objetivo la enumeración de todas las especies de plantas, animales, hongos y microbios de la Tierra para su uso en la amplia variedad de contextos relacionados con la biodiversidad global.

UNEP Global Resources Information Database (GRID): http://grid2.cr.usgs.gov/

Forma parte de la red Global Environment Monitoring System (GEMS) y tiene como objetivo colectar y difundir la información más avanzada sobre el estado mundial de los recursos naturales. La información se organiza en forma de datos georreferenciados procedentes de varias fuentes incluyendo las de la red GEMS.

UNEP International Environmental Information System (INFOTERRA):

http://www.cedar.univie.ac.at/unep/infoterral

INFOTERRA se creó para facilitar el intercambio de información ambiental entre naciones. La información es disponible a través de medios variados, incluyendo publicaciones regulares tales como International Directory of Sources y World Directory of Environmental Expertise.

UNEP-United Nations Environment Programme: http://www.unep.ch

Este web es mantenido por UNEP's Information Unit for Conventions (IUC) para hacer más asequible la información del centro ejecutivo de Ginebra sobre tratados y programas de medio ambiente. Además proporciona direcciones a otros sitios relacionados.

UNEP/Convention on International Trade in Endangered Species of Wild Fauna and Flora (CITES) Secretariat:

http://www.unep.ch/cites.html

En estas páginas se encuentra toda la información relativa al convenio CITES

United Nations Educational, Scientific and Cultural Organization (UNESCO):

http://www.unesco.org/

Tiene establecidas varias formas de cooperación internacional relacionadas con las ciencias ambientales e investigación sobre recursos naturales, con programas clave que incluyen el programa The Man and the Biosphere, la International Network of Biosphere Reserves y el World Heritage Convention UNESCO-Ecological sciences and the Man and the Biosphere (MAB) programme:

http://www.unesco.org/general/eng/programmes/ science/programme/environ/mab.html

El programa $\mathrm{MAB}$ es un esfuerzo multidisciplinar para combinar las ciencias naturales y sociales, ayudar a controlar los conflictos entre el ambiente y el desarrollo en la biosfera.

World Conservation Monitoring Centre (WCMC): http://www.wcmc.org.uk/

Proporciona servicios de información sobre conservación y uso sostenible de los recursos biológicos y ayuda a otros a desarrollar sus propios servicios de información.

World Species List Taxa Database Plants Animals Microbes (WSL):

http://envirolink.org/species/

Este servidor pretende realizar una lista, no centralizada, de todas las especies del mundo. Las listas son mantenidas por los contribuyentes que las proporcionan.

ORGANIZACIONES EUROPEAS

European Center for Nature Conservation (ECNC): http://www.ecnc.nl/

Red de institutos de conservación de la naturaleza de Europa y de organismos de conservación internacionales. 


\section{European Environment Agency (EEA):}

http://www.eea.dk/

La Agencia Europea de Medioambiente (EEA) fue concebida como una unidad de coordinación para una gran red descentraliza de la Unión Europea y sus estados miembros, creada para proporcionar información estratégica de relevancia y una valoración sobre el estado del medioambiente en Europa. Dentro de las ocho áreas que trata se encuentra la protección de la fauna y flora que está coordinada por el Centro Temático Europeo para la Conservación de la Naturaleza (ETC/NC)

European Science Foundation ESF Network in Systematic Biology: http://www.nhm.ac.uk/esf/

El objetivo de esta red es apoyar la biología sistemática promoviendo la comunicación entre sistemáticos en Europa.

European Topic Center on Nature Conservation

(ETC/NC): http://www.mnhn.fr/ctn/

El Centro Temático Europeo para la Conservación de la Naturaleza (ETC/NC) fue creado por la Agencia Europea de Medioambiente (EEA) y está integrado por 15 instituciones de 12 países y cada una de las cuales se ocupa de una tarea específica dentro de este campo.

\section{ORgANiZACIONES NACIONALES}

Australian Environment Online:

http://www.erin.gov.au/

Presenta un amplio abanico de información sobre biodiversidad en Australia y alberga un gran número de bases de datos nacionales.

Base de Dados Tropical: http://www.bdt.org.br/

Es un departamento de la Fundação Tropical de Pesquisas e Tecnologia "André Tosello", fundación privada sin ánimo de lucro. Colabora con el secretariado de la Convención sobre Diversidad Biológica en Montreal y con un buen número de organizaciones. Mantiene un gran número de bases de datos nacionales sobre biodiversidad y otros recursos de Internet.

Biodiversity and Ecosystems NEtwork (BENE): http://straylight.tamu.edu/bene/bene.html Canadian Biodiversity Information Network (CBIN): http://www.doe.ca/ecs/biodiv/biodiv.html Chinese Biodiversity Information System (CBIS): http://panda.ioz.ac.cn/brim/cbis.html Comision Nacional para el Conocimiento y Uso de la Biodiversidad (CONABIO):

http://www.conabio.gob.mx/

CostaRican Conservation Data Centre at the Fundación Neotropica

INBio: $h t t p: / / w w w . i n b i o . a c . c r / e s p a n o l . h t m l$
Es una institución que se concentra única $\mathrm{y}$ exclusivamente en la realización de actividades de investigación y conservación de la biodiversidad en forma integrada, con un enfoque pragmático y una nueva meta claramente definida: poner la biodiversidad a trabajar de manera sostenida para la sociedad costarricense.

SERVICIOS DE INFORMACIÓN SOBRE ORGANIZACIONES Biodiversity Resource Center:

http://www.biodiv.com/rcenters/biodiv/biodiv.html Directory of Nature Conservation Organizations: http://www.ecnc.nl/doc/europe/organiza/director.html

IRRO Directory to Information Resources:

http://www.bdt.org.br/bdt/irro/irrodir

List of Environmental Resources:

http://www.runet.edu/\%7Egeog-web/EnvTopics.html

National Library for the Environment from

CNIE*: http://www.cnie.org/nle/

Environment Related Websites:

http://www.envision.net/osites/environ/envrelat.html

Zoological and other related bioscience organizations:

http://www.york.biosis.org/zrdocs/zoolinfo/zoolorgs.htm

Biodiversity and Biological Collections WWW

Server: http://www.keil.ukans.edu/

Eco-Network / Coolest Environmental 100!:

http://www.compulink.gr/eco-network/hyperlinks.html

\section{AGRADECIMIENTOS}

Los autores agradecen a Isabel Morón la revisión crítica del apartado de documentación bibliográfica.

\section{Referencias}

ANÓNIMO, 1997. Motores de búsqueda. PC-MEDIA, 32: 82-88.

CARling, R.C.J. \& HARrison, J., 1996. Biodiversity information on the Internet: cornucopia or confusion?. BiodiversityLetters, 3: 125-135.

FARR, D.F. \& Rossman, A.Y., 1997. Integration of data for biodiversity initiatives. In: M.L. Reaka-Kudla, D.E. Wilson \& E.O. Wilson (eds.). Biodiversity II. Understanding and Protecting our Biological Resources. Joseph Henry Press, Washington D. C.: 475-490.

NiElsEN, C., 1995. Animal Evolution, Oxford University Press, Oxford.

Olivieri, S.T., Harrison, J. \& Busby, J.R., 1995. Data and Information Management and Communication. In: V.H. Heywood \& R.T.Watson (eds.). Global 
Biodiversity Assessment. United Nations Environment Programme. Cambridge Univ. Press, Cambridge: 609-670.

OscA-Lluch, J., 1997. Aproximación a una bibliografía española sobre Internet. Boletín de la ANABAD, 47(1): 101-106.
SÁnchez-Biezma, N. \& Aguillo, I.F., 1997. Biología marina en la red. Mundo Científico, 184: 931-949.

Publicado, el 30-VI-1998 